\title{
Reduktion von Metallartefakten in der muskuloskelettalen Bildgebung
}

\section{Reduction of metal artefacts in musculoskeletal imaging}

\author{
R. Sutter, T. Dietrich
}

\section{Übersicht}

Einleitung

Metallartefakte in der MRT

Metallartefakte in der CT

Klinische Bedeutung der MRT und $C T$

\section{Zusammenfassung}

Gelenkprothesen und andere orthopädische Implantate werden bei vielen Patienten mit muskuloskelettalen Erkrankungen eingesetzt. Während diese Operationen häufig ein gutes klini-

\section{Einleitung}

Gelenkprothesen an Hüfte, Knie und anderen Gelenken werden heute bereits sehr häufig eingesetzt und aus demografischen Gründen wird ihre Zahl in den nächsten 2 Jahrzehnten weiter ansteigen [1]. Computertomografie (CT) und Magnetresonanztomografie (MRT) sind 2 wichtige Modalitäten für die muskuloskelettale Bildgebung und werden oft bei Patienten mit Metallimplantaten eingesetzt [2]. Implantate führen jedoch zu multiplen Artefakten in CT und MRT mit teilweise stark eingeschränkter Bildqualität. Besonders in der muskuloskelettalen Bildgebung hat dies wichtige Konsequenzen.

Während das eigentliche Untersuchungsgebiet bei einem Patienten mit einer Hüft-TEP in der abdominalen Bildgebung vergleichsweise weit vom Metallimplantat entfernt ist, untersucht man in der muskuloskelettalen Bildgebung i.d.R. die anatomische Gegend direkt im Umfeld der Prothese, beispielsweise um postoperative Komplikationen oder unklare Beschwerden abzuklären. Somit ist eine leistungsstarke Reduktion von Metallartefakten in der muskuloskelet- sches Ergebnis zeigen, sind im Verlauf bei vielen Patienten radiologische Untersuchungen notwendig. Orthopädische Implantate führen jedoch in der MRT und der CT zu starken Metallartefakten. Dieser Artikel stellt mehrere grundlegende Methoden sowie fortgeschrittene Techniken zur Reduktion dieser Artefakte für MRT und CT vor, um eine diagnostische Untersuchung bei Patienten mit Metallimplantaten zu ermöglichen. MRT und CT werden so zu wichtigen und zuverlässigen Modalitäten, um Patienten mit Gelenkprothesen und orthopädischen Implantaten zu untersuchen. talen Bildgebung im Vergleich zu anderen radiologischen Subspezialitäten von besonderer Bedeutung.

Nachfolgend werden Grundprinzipien und fortgeschrittene Techniken der Reduktion von Metallartefakten in der CT und der MRT erläutert und ihre klinische Bedeutung illustriert.

\section{Metallartefakte in der MRT}

\section{Entstehung der Artefakte}

Magnetische Suszeptibilität. Der menschliche Körper besteht aus einer Vielzahl von Gewebearten, die eine unterschiedliche Magnetisierbarkeit, die sog. magnetische Suszeptibilität aufweisen [3]. Dabei wird zwischen dia-, para- und ferromagnetischen Substanzen unterschieden (Tab. 1). Diamagnetische Substanzen, die im menschlichen Körper am häufigsten vorkommen, schwächen ein von außen einwirkendes Magnetfeld leicht ab, paramagnetische Substanzen verstärken es und ferromagnetische Substanzen wie Eisen oder Kobalt verstärken es beträchtlich, was zu starken Ver- 


\section{Tabelle 1}

Magnetische Suszeptibilität.

\begin{tabular}{|lll|}
\hline Substanzklasse & Beispiele & Effekt auf Magnetfeld \\
\hline diamagnetisch & $\begin{array}{l}\text { Kalzium, Wasser, meiste } \\
\text { organische Substanzen }\end{array}$ & leichte Abschwächung \\
\hline paramagnetisch & $\begin{array}{l}\text { Titan, Luft, Gadolinium- } \\
\text { Kontrastmittel, einzelne } \\
\text { Blutabbauprodukte }\end{array}$ & leichte Verstärkung \\
\hline ferromagnetisch & $\begin{array}{l}\text { Eisen, Kobalt-Chrom, } \\
\text { Nickel }\end{array}$ & $\begin{array}{l}\text { deutliche Verstärkung mit resul- } \\
\text { tierend starker Verzerrung }\end{array}$ \\
\hline
\end{tabular}
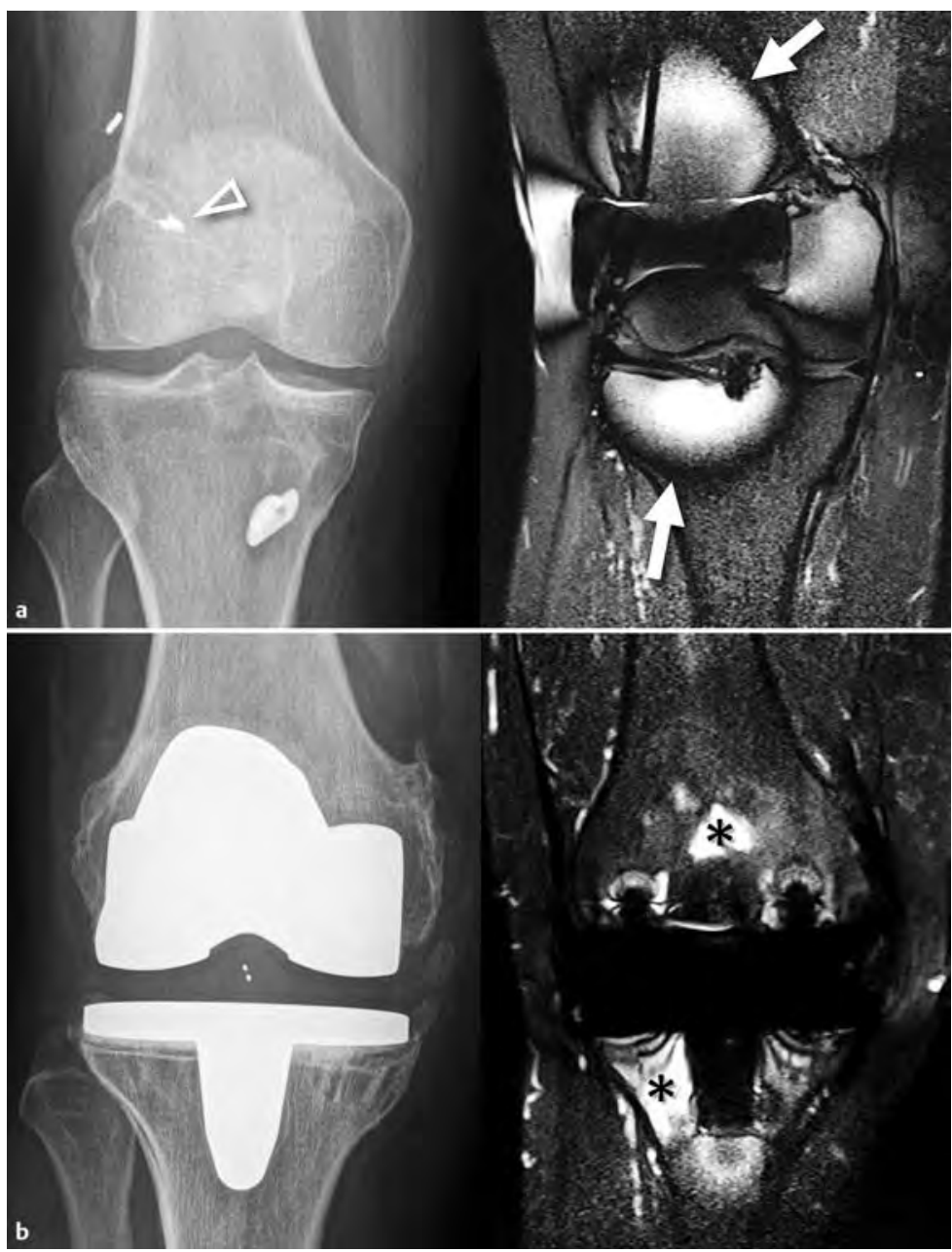

Abb. 1 Einfluss der magnetischen Suszeptibilität auf das Ausmaß der Metallartefakte in der MRT. a Bei Status nach Kreuzbandplastik ist eine abgebrochene Bohrerspitze aus Edelstahl (Pfeilspitze) im distalen Femur auf dem Röntgenbild abgrenzbar. Im korrespondierenden koronaren MRT-Bild sind schwere Metallartefakte mit Kleeblatt-Morphologie (Pfeile) vorhanden, da das Material der Bohrerspitze stark ferromagnetisch ist. b Nach Implantation einer Knieprothese ist zwar viel mehr Metall am Kniegelenk vorhanden - hier wurde jedoch Titan als Material verwendet. Dieses ist paramagnetisch, womit sehr viel geringere Artefakte im koronaren MRT-Bild vorhanden sind und sogar Osteolysezonen $\left(^{*}\right)$ angrenzend an das Metall abgrenzbar sind. zerrungen des Magnetfeldes und damit einhergehenden schweren Bildartefakten führt.

Am stärksten wird das Magnetfeld durch ferromagnetische Substanzen wie Eisen oder Kobalt verändert.

Implantatmaterial. Während ein Teil der modernen medizinischen Implantate aus Titan besteht, werden auch noch heute viele ferromagnetische Implantate verwendet, z. B. Kobalt-Chrom-Legierungen, die mit entsprechend starken Artefakten in der MRT einhergehen. Auch Bohrer aus rostfreiem Stahl sind häufig ferromagnetisch. Wenn eine Bohrerspitze während einer Operation abbricht und nicht aus dem Knochen entfernt werden kann, wird dies zum Problem bei anschließenden MRT-Untersuchungen, da durch das ferromagnetische Material starke Metallartefakte im Bild entstehen (Abb.1).

Implantatgeometrie. Eine weitere Rolle spielt die Geometrie des verwendeten Implantates. Eine einfache Plattenosteosynthese führt beispielsweise zu weniger Implantaten als eine Gelenkprothese.

Magnetfelder. Während einer MRT-Untersuchung sind viele Magnetfelder vorhanden, die durch Metallimplantate gestört werden können [4]:

- das statische Magnetfeld $\left(\mathrm{B}_{0}\right)$, das konstant im Gerät vorhanden ist

- der elektromagnetische Anregungspuls $\left(B_{1}\right)$, der durch die Radiofrequenzspule generiert wird

- die Gradienten-Magnetfelder in den 3 Ebenen des Raums $\left(G_{x}, G_{y}, G_{z}\right)$, welche die selektive Anregung einer Körperregion ermöglichen und die räumliche Information des Signals codieren

Metallimplantate führen zu Verzerrungen in allen diesen Magnetfeldern mit entsprechend teils stark reduzierter Bildqualität.

\section{Typen der Artefakte}

Die Metallartefakte in der MRT können in verschiedene Grundtypen eingeteilt werden [3]:

Signalverlust und -stapelung. Ein Signalverlust entsteht z. B. aufgrund der Dephasierung von Spins im Rahmen der großen Frequenzvariationen des Magnetfeldes, während sich das Bildsignal bei der Signalstapelung aus verschiedenen Arealen an einer falschen Stelle kumulieren kann. 
Geometrische Distorsion. Bei der geometrischen Distorsion wird ein Voxel mit einer bestimmten Bildinformation einer inkorrekten anatomischen Lokalisation zugewiesen, was zu Verzerrungen im erhaltenen Bild führt.

Fettunterdrückung. Besonders anfällig sind zudem Sequenzen mit frequenzselektiver Fettunterdrückung. Eine solche ist bei Patienten mit Metallimplantaten häufig ungenügend, was sich in einer fehlenden oder inhomogenen Fettunterdrückung in bestimmten Bildarealen zeigt (Abb.2), wobei die Areale direkt um das Metallimplantat besonders beeinträchtigt sind.

Ursprungsbildebene. Zudem werden Metallartefakte, die aus der Bildebene selbst stammen, von solchen Metallartefakten unterschieden, welche aus benachbarten Ebenen auf die Bildebene einwirken. Während herkömmliche Strategien zur Artefaktkorrektur vor allem die Artefakte aus derselben Bildebene korrigieren, ist mittels fortgeschrittener MRT-Sequenzen auch die zusätzliche Artefaktkorrektur aus den benachbarten Ebenen möglich.

\section{Einfache Artefaktkorrektur}

Bei den Methoden zur Artefaktkorrektur wird zwischen einfachen und fortgeschrittenen Verfahren unterschieden (Tab.2).

\section{Gerätewahl: 1,5T vs. 3T}

Metallartefakte sind direkt proportional zur Stärke des Magnetfeldes, was sich in einer deutlich schlechteren Bildqualität bei $3 \mathrm{~T}$ im Vergleich zu 1,5T zeigt (Abb.3). Auch bei $3 \mathrm{~T}$ gibt es eine Vielzahl von Techniken, um Metallartefakte zu reduzieren, jedoch startet man auf einem massiv höheren Artefaktniveau und muss deutlich potentere Techniken einsetzen, um eine ähnliche Bildqualität wie bei 1,5T zu erreichen. Daher sollte man Patienten mit Metallimplantaten bei 1,5T untersuchen.

Je stärker das Magnetfeld, desto ausgeprägter sind die Metallartefakte.

\section{Erhöhung der Bandbreite}

Empfängerbandbreite. Die Erhöhung der Empfängerbandbreite ermöglicht es zwar, die Signalverschiebungen in der Ausleserichtung zu reduzieren, geht aber mit einer verminderten Effizienz des Signal-zu-RauschVerhältnisses (SNR) einher [3]. Erhöht man die Empfängerbandbreite um $100 \mathrm{~Hz} /$ Pixel auf $400 \mathrm{~Hz} /$ Pixel, reduziert dies bereits die Artefakte. Empfehlenswert

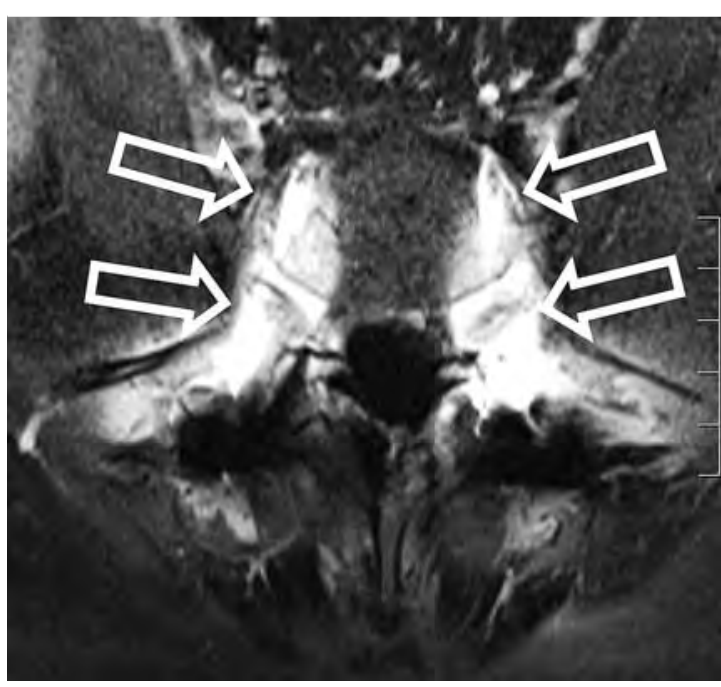

Abb. 2 Die spek trale Fettsaturierung ist sehr anfällig für Metallartefakte. Axiale T1w fettsatu rierte MRT-Sequenz der Lendenwirbelsäule mit Versagen der Fettunterdrückung (Pfeile) bei einem Patienten mit Spondylodese.

\section{Tabelle 2}

Artefaktkorrektur in der MRT.

\begin{tabular}{|ll|}
\hline Technik & Limitation \\
\hline Einfache Techniken & \\
\hline Erhöhung der Empfängerbandbreite & $\begin{array}{l}\text { beschränkter Effekt, insbesondere bei } \\
\text { Fettsaturierung }\end{array}$ \\
\hline 1,5T- statt 3T-Gerät verwenden & logistische Verfügbarkeit \\
\hline dünne Schichten & niedriges SNR, lange Akquisitionszeit \\
\hline $\begin{array}{l}\text { Einsatz von Turbo-Spin-Echo- } \\
\text { Sequenzen }\end{array}$ & $\begin{array}{l}\text { beschränkter Effekt, insbesondere bei } \\
\text { Fettsaturierung }\end{array}$ \\
\hline STIR statt spektrale Fettsaturation & niedriges SNR, versagt bei Gelenkprothese \\
\hline Fortgeschrittene Techniken & Bildunschärfe \\
\hline STIR mit optimiertem Inversionspuls & versagt direkt an Metall-Knochen-Grenze \\
\hline STIR WARP) & \\
\hline
\end{tabular}

ist es jedoch, die Empfängerbandbreite insbesondere bei der MRT von Patienten mit Gelenkprothesen auf Werte bis $500-800 \mathrm{~Hz} /$ Pixel zu erhöhen. Allerdings stößt man mit dieser Methode zur Artefaktkorrektur rasch an Grenzen - insbesondere bei ferromagnetischen Materialien oder bei fettsaturierten Sequenzen sind meist starke Restartefakte vorhanden. 


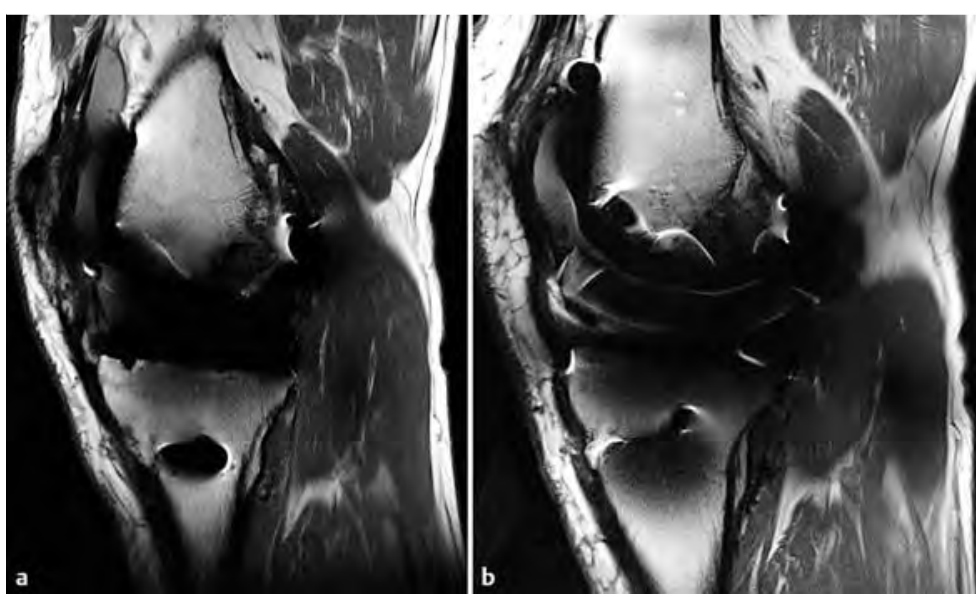

Abb. 3 Einfluss der Feldstärke auf die Metallartefakte: Sagittale protonengewichtete Sequenz mit erhöhter Empfängerbandbreite bei Patienten mit Knie-TEP im 1,5T-MRT (a) und 3T-MRT (b). Bei 3 T sind deutlich größere Artefakte vorhanden.
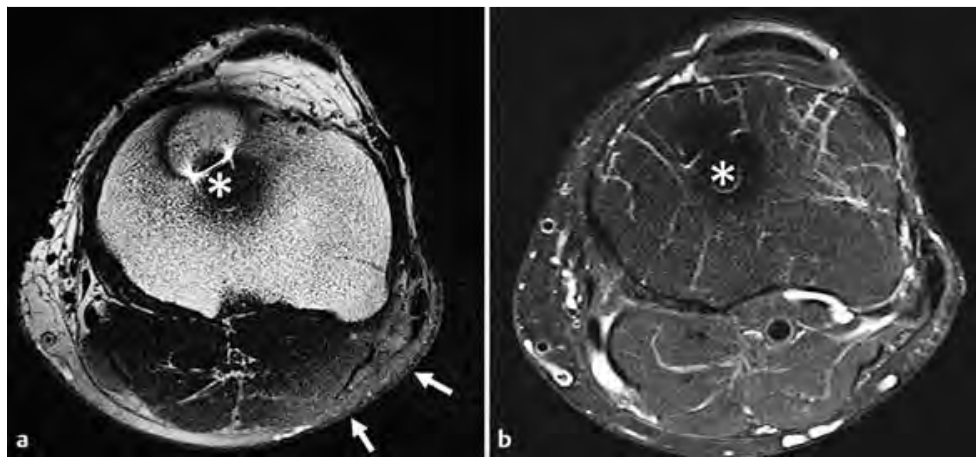

Abb. 4 Patient mit Ligamys-Schraube $\left({ }^{*}\right)$ zentral im Tibiakopf nach Kreuzbandplastik. a In der axialen protonengewichteten Sequenz mit spektraler Fettsaturierung ist ein fast vollständiges Versagen der Fettunterdrückung sichtbar. Lediglich im posterolateralen Aspekt des Bildes (Pfeile) sind Areale mit guter Fettunterdrückung sichtbar. b In der axialen STIRSequenz mit optimiertem Inversionspuls (STIR WARP) ist hingegen eine gute Fettunterdrückung über das gesamte Bild vorhanden.

Für Patienten mit Gelenkprothesen sollte die Empfängerbandbreite auf Werte bis $500-800 \mathrm{~Hz} /$ Pixel erhöht werden.

Bandbreite des Anregungspulses. Auch die Bandbreite des Anregungspulses kann erhöht werden, um Metallartefakte zu reduzieren. Diese Technik ist in einzelnen der modernen Sequenzen als Bestandteil zur Artefaktreduktion enthalten, kann jedoch teilweise auch als separate Technik eingesetzt werden.

\section{- Schichtdicke und Voxelgröße}

Das Verwenden dünnerer Schichten erlaubt es, einen Teil der Bildartefakte aus benachbarten Schichten zu verringern. Auch eine Verkleinerung der Voxelgröße durch Erhöhen der Bildmatrix trägt infolge einer Verminderung der Spin-Dephasierung innerhalb des Voxels zu einer Reduktion der Metallartefakte bei.
Sowohl dünnere Schichten als auch eine kleinere Voxelgröße sind jedoch mit einer längeren Messzeit und einer SNR-Reduktion verbunden. Zudem wird die anatomische Abdeckung der zu untersuchenden Region limitiert. Deshalb empfiehlt sich zur vollständigen Darstellung auch der Peripherie des Untersuchungsgebietes z. B. der Einsatz von einzelnen Sequenzen mit dünnen Schichten in Kombination mit anderen Sequenzen, die eine größere Schichtdicke aufweisen am besten wird dies kombiniert mit weiteren Methoden der Artefaktreduktion.

\section{Einsatz von Turbo-Spin-Echo-Sequenzen}

Während Gradienten-Echo-Sequenzen für eine Vielzahl von Anwendungen in der muskuloskelettalen Bildgebung vorteilhaft sind, werden sie in der Metallbildgebung nicht eingesetzt, da sie aufgrund der ausgeprägten Artefakte oft nicht diagnostisch sind. Hingegen sind Turbo-Spin-Echo-Sequenzen gut geeignet, um Metallartefakte zu reduzieren, da diese Sequenzen multiple $180^{\circ}$-Refokussierungspulse aufweisen, was zu einer Verringerung der durch die Feldverzerrung verursachten Dephasierung der Spins führt.

Gradienten-Echo-Sequenzen sollten in der Metallbildgebung nicht eingesetzt werden.

\section{Fettunterdrückung}

Flüssigkeitssensitive Sequenzen werden in der muskuloskelettalen Radiologie sehr häufig eingesetzt, um pathologische Befunde im Knochen und in den Weichteilen zu beurteilen. Allerdings ist die Fettunterdrückung bei Metallimplantaten vielfach ungenügend [3], insbesondere wenn eine spektrale Fettsaturierung verwendet wird (Abb.4). Bei der spektralen Fettsaturierung macht man sich die unterschiedliche Resonanzfrequenz der fett- und wassergebundenen Protonen zunutze: Ein zusätzlich angelegter Radiofrequenzpuls unterdrückt hier normalerweise das Fettsignal. Bei metallinduzierten Verzerrungen im Magnetfeld reicht der angelegte Radiofrequenzpuls jedoch nicht aus, um das Fettsignal adäquat zu unterdrücken und es resultiert eine inhomogene Fettsaturation.

Eine Möglichkeit, um in der Metallbildgebung eine homogene Fettunterdrückung zu erreichen, ist das Verwenden von STIR-Sequenzen. Im Gegensatz zur spektralen Fettsaturierung basiert die STIR-Sequenz nicht auf Frequenzunterschieden, sondern auf der unterschiedlichen T1-Relaxationszeit von Fett und Wasser. Durch Anlegen eines $180^{\circ}$-Inversionspulses wird die longitudinale Magnetisierung sowohl der fettals auch der wassergebundenen Protonen invertiert. Da 
Fett eine deutlich kürzere Relaxationszeit als Wasser aufweist, kann die Anregung der wassergebundenen Protonen just zu dem Zeitpunkt durchgeführt werden, an dem die fettgebundenen Protonen von der negativen zur positiven longitudinalen Magnetisierung wechseln und somit einen Wert von 0 aufweisen. Es resultiert eine selektive Anregung wassergebundener Protonen, die auch bei Patienten mit Metallimplantaten recht zuverlässig funktioniert, insbesondere einige Zentimeter vom Metall entfernt. Eine Kombination von STIR-Sequenz und erhöhter Bandbreite potenziert die Reduktion der Metallartefakte, wobei die Methode jedoch in Arealen, die direkt an das Metall angrenzen, nur eingeschränkt oder gar nicht mehr funktioniert.

\section{Fortgeschrittene Techniken}

Seit einigen Jahren sind neuere Sequenzen zur Reduktion von Metallartefakten verfügbar, die teilweise spezifisch für diesen Zweck entwickelt oder optimiert wurden. Diese sind mit einem erhöhten technischen Aufwand verbunden und müssen $z$. T. separat vom Gerätehersteller erworben werden. Die wichtigsten dieser neueren Techniken werden im Folgenden vorgestellt.

\section{- STIR mit optimiertem Inversionspuls}

Wenn der Inversions- und der Anregungspuls unterschiedliche Bandbreiten aufweisen, ist das Ausmaß der Verzerrung der beiden Pulse unterschiedlich und die Fettunterdrückung folglich ungenügend. Dies kann man verhindern, indem man für beide Pulse die identische Bandbreite verwendet und hierfür eine hohe Bandbreite wählt (Abb.5). Während die räumliche Verzerrung dadurch zwar nur mäßig reduziert wird, hat der Einsatz der STIR-Sequenz mit optimiertem Inversionspuls (sog. STIR WARP) einen guten Effekt auf die Homogenität der Fettunterdrückung [5]. Dies zeigt sich insbesondere in einem deutlich größeren Areal mit korrekter Fettunterdrückung als bei Verwendung einer Standard-STIR-Sequenz. Zudem funktioniert die STIRSequenz mit optimiertem Inversionspuls auch bei kurzen Akquisitionszeiten recht stabil.

Die STIR-Sequenz mit optimiertem Inversionspuls ist vielseitig in der klinischen MRT-Diagnostik einsetzbar und führt bei den meisten Implantaten zu einer homogenen Fettunterdrückung.

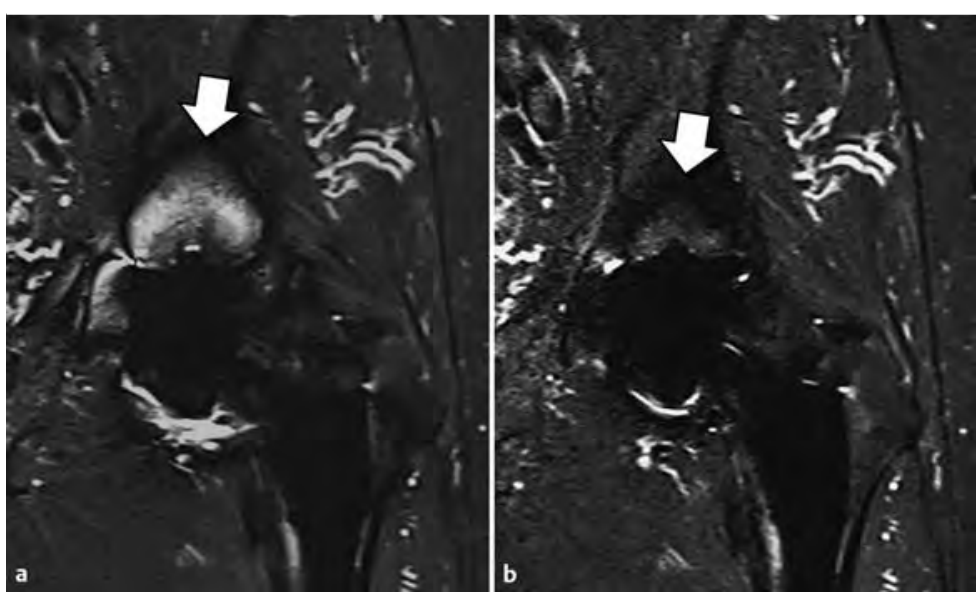

Abb. 5 Methoden zur MRT-Artefaktreduktion bei Fettunterdrückung und implantierter Hüft-TEP. a Bereits mit Erhöhung der Empfängerbandbreite kann häufig eine diagnostische Bildqualität erreicht werden. Wie diese koronare STIR-Sequenz mit erhöhter Empfängerbandbreite zeigt, sind jedoch mit dieser Methode mehrere Areale mit ungenügender Fettunterdrückung (Pfeil) um die Prothesenpfanne vorhanden. b Wenn zusätzlich ein optimierter STIR-Inversionspuls angewendet wird (STIR WARP), sind zwar noch Artefaktareale um die Prothesenpfanne abgrenzbar (Pfeil), jedoch deutlich kleiner.

\section{Dixon-Technik}

Ähnlich wie die spektrale Fettsaturierung basiert auch die Dixon-Sequenz auf einer unterschiedlichen Resonanzfrequenz von fett- und wassergebundenen Protonen. Eine Fettunterdrückung wird hier jedoch direkt auf molekularer Ebene erreicht, da die Technik darauf basiert, dass die Spins von fett- und wassergebundenen Protonen eine unterschiedliche Präzessionsfrequenz aufweisen [6]. Somit sind zu bestimmten Zeitpunkten die Fett- und Wasser-Spins gleichgerichtet (engl. „inphase“) oder entgegenwirkend („opposed-phase“). Diese beiden Phasen können gemessen werden, und anschließend werden daraus ein Fett-Bild und ein Wasser-Bild errechnet. Diese Technik ist insgesamt zwar wenig anfällig auf Inhomogenitäten des Hauptmagnetfeldes und des Anregungspulses, versagt jedoch typischerweise direkt am Metall-Gewebe-Übergang und zieht auch eine verlängerte Bildakquisition und Bildrekonstruktion nach sich (Abb.6). Ein Vorteil der Dixon-Technik ist jedoch ein besseres SNR sowie eine höhere Auflösung als mit der STIR-Technik.

\section{View-Angle-Tilting (VAT)}

Beim View-Angle-Tilting (VAT) wird während des Auslesens des MRT-Signals ein zusätzlicher Kompensationsgradient eingeschaltet, der den Winkel des Auslesens dreht. Die VAT-induzierte Verzerrung kompensiert hierbei jene durch das Metall induzierte Verzerrung oder hebt diese zumindest teilweise auf [3]. 

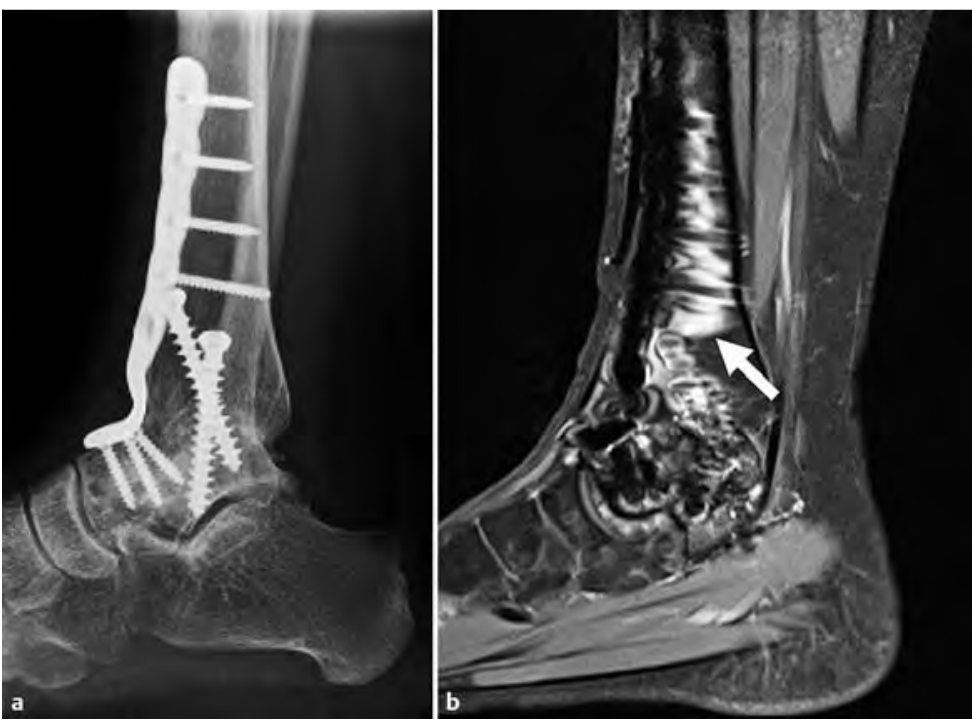

Abb.6 Patient mit breitflächig durchbauter Arthrodese des oberen Sprunggelenks. a Im seitlichen Röntgenbild sind eine Platte und multiple Osteosyntheseschrauben sichtbar. b Mittels Dixon-Technik (hier mit T1-Gewichtung nach i.v. Kontrastmittelapplikation) wurde eine sagittale Sequenz akquiriert, von welcher hier das Wasserbild wiedergegeben wird. Mittels Dixon-Technik sind direkt um das Osteosynthesematerial teils deutliche Restartefakte vorhanden (Pfeil), jedoch weist der Rest des Bildes eine gute und homogene Fettunterdrückung auf.

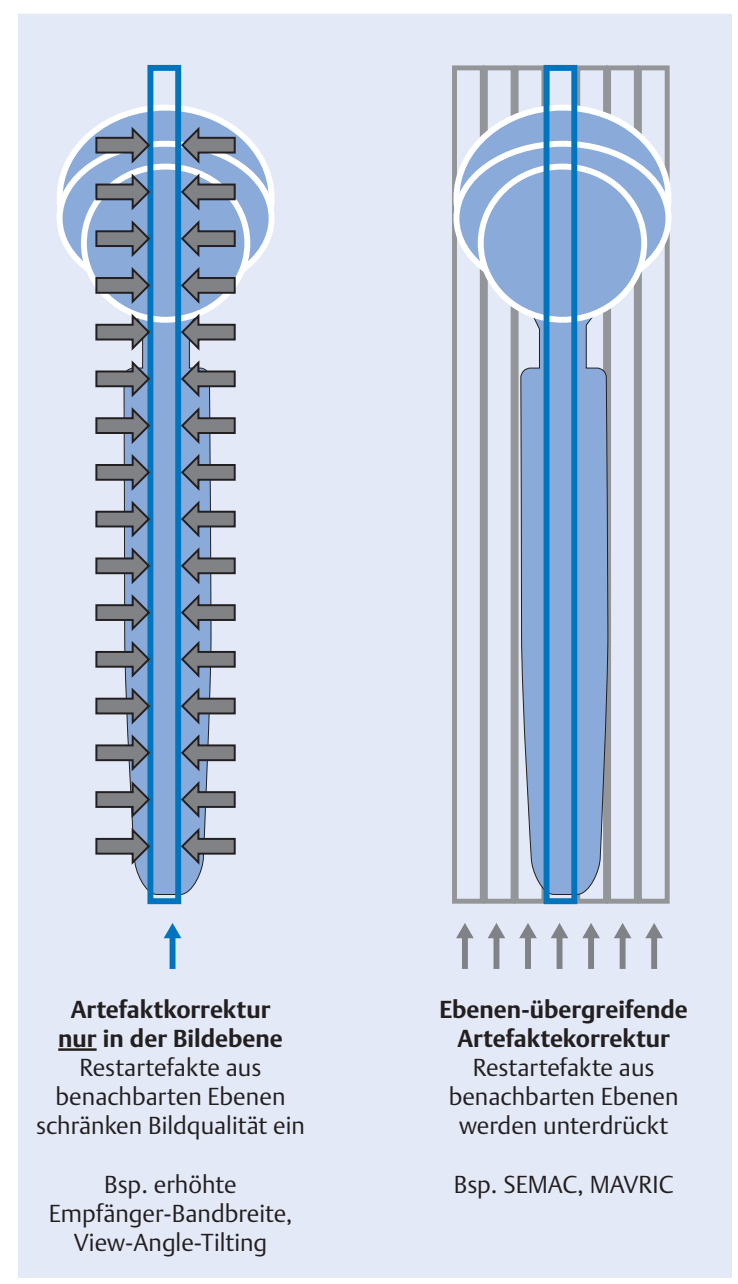

Allerdings kommt es durch das VAT an den Grenzstellen zwischen verschiedenen Gewebearten zu einer teils deutlichen Bildunschärfe, je nachdem, welche räumliche Orientierung diese aufweisen. Deshalb muss das Ausmaß der VAT-Korrektur sorgfältig mit dem Bildeindruck abgewogen werden.

\section{- Ebenen-übergreifende Artefaktkorrektur}

Multi-spektrale Techniken wie „Slice encoding for metal artifact correction“ (SEMAC) und „Multi-acquisition variable-resonance image combination" (MAVRIC) messen für jede Bildschicht ein dreidimensionales Volumen (Abb.7).

Die multispektralen Techniken erlauben es, die aus den benachbarten Ebenen auf die Bildebene einwirkenden Metallartefakte zu messen und zu unterdrücken (sog. Through-Plane-Correction).

SEMAC. SEMAC basiert auf einer Spin-Echo-Sequenz, die eine zusätzliche Phasencodierung entlang der Schichtselektion durchführt [7]. Bildschichten, die durch ein Metallimplantat dreidimensional verzerrt sind (ähnlich der Wölbung von Kartoffelchips), können
Abb.7 Schematische Darstellung der Artefaktkorrektur nur innerhalb der Bildebene (links) und der ebenenübergreifenden Artefaktkorrektur (rechts). Bei der ersten Methode wirken noch deutliche Artefakte aus benachbarten Ebenen auf die Bildebene ein (links). Diese Artefakte werden bei der zweiten Methode (rechts) für jedes einzelne Bild mit einem dreidimensionalen Datensatz gemessen und bei der Bildkorrektur unterdrückt.

so in eine flache Form zurücktransformiert werden, die der korrekten anatomischen Darstellung entspricht (Abb.8). Um gleichzeitig die Artefakte innerhalb der Bildebene zu korrigieren, wird SEMAC häufig in Kombination mit VAT verwendet (Abb.9).

MAVRIC. Bei MAVRIC hingegen werden dreidimensionale Messungen mit multiplen überlappenden Frequenzen akquiriert, die anschließend zusammengerechnet werden, um ein artefaktkorrigiertes Bild zu erzeugen [8].

Nachteil. Ein Nachteil von SEMAC und MAVRIC im klinischen Einsatz ist die teils deutlich verlängerte Akquisitionszeit von 6-8 Minuten, abhängig davon, wie stark die Artefakte korrigiert werden sollen. Die 


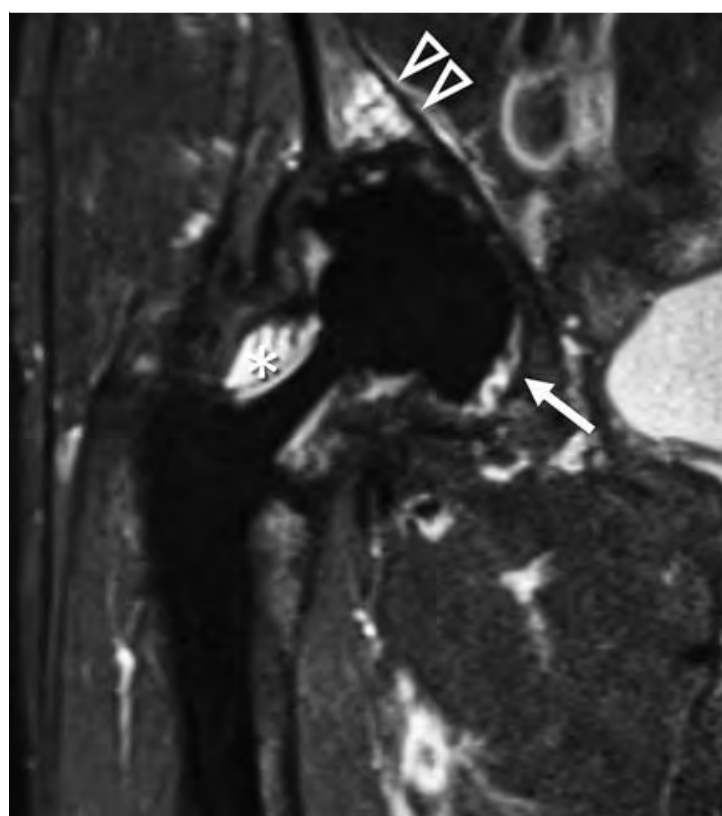

Abb. 8 Pfannenlockerung bei Hüft-TEP mit Polyethylenabrieb. Ein koronares STIR-Bild zeigt den potenten Einsatz der SEMAC-Technik zur ebenenübergreifenden Artefaktkorrektur. Um das Prothesenmaterial sind beinahe keine Restartefakte mehr sichtbar. Gut dargestellt sind hingegen die Synovitis ( $\left.{ }^{*}\right)$ im Hüftgelenk, der Osteolysesaum um die Prothesenpfanne (Pfeil) und ein fokales Knochenmarködem kranial angrenzend an die Pfanne (Pfeilspitzen).

SEMAC- und MAVRIC-Technik können gekoppelt werden, um eine noch stärkere Artefaktkorrektur zu erreichen. Diese Sequenz wird als MAVRIC-SL bezeichnet [8].

\section{Perspektiven}

\section{Compressed Sensing}

Typischerweise benötigen Sequenzen zur ebenenübergreifenden Artefaktkorrektur wie SEMAC oder MAVRIC eine lange Akquisitionszeit. Diese kann jedoch deutlich verkürzt werden, wenn die Anzahl der außerhalb der Bildebene erhobenen Messpunkte reduziert wird.

Die Verkürzung der Akquisitionszeit basiert auf dem mathematischen Konzept „Sparsity“ bzw. „Compressed Sensing“: In einer relativ schnellen Bildakquisition wird ein niedrig aufgelöstes Datenvolumen gemessen, um daraus mittels iterativer Nachrekonstruktion ein hochaufgelöstes Bild zu erhalten [10].

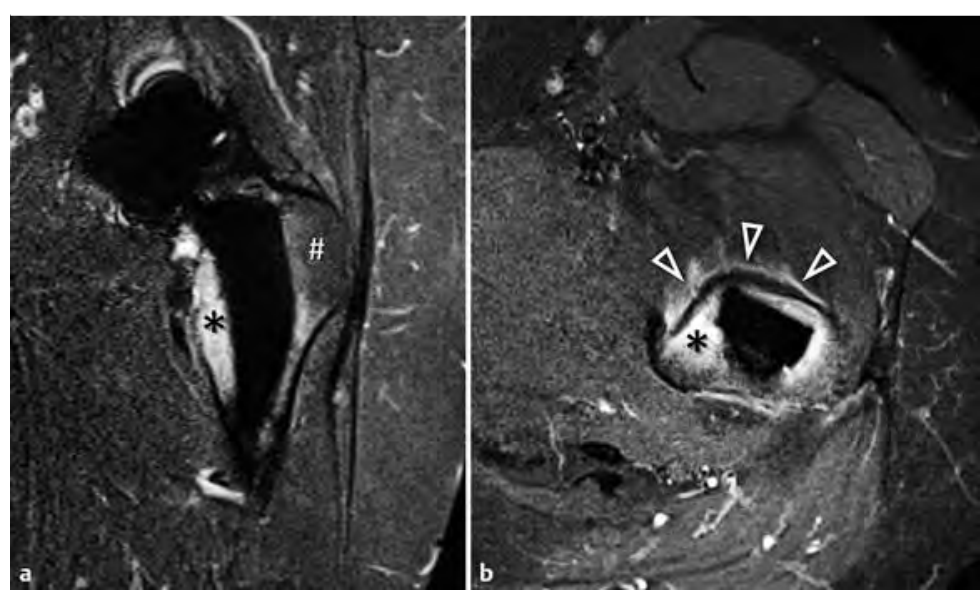

Abb.9 Stressreaktion nach Hüft-TEP. a Die koronare STIR-SEMAC-Sequenz zeigt ein Knochenmarködem $\left({ }^{*}\right)$ am proximalen Femurschaft, das sich deutlich vom normalen Knochenmarksignal (\#) auf Höhe der Prothesenschulter unterscheidet. b Die axiale STIRSequenz mit optimiertem Inversionspuls (STIR WARP) auf Höhe des proximalen Femurschaftes zeigt Ödemzonen sowohl im Knochenmark $\left({ }^{*}\right)$ als auch ventral am Periost (Pfeilspitzen).

Bei SEMAC beispielsweise ist das relevante Signal in den benachbarten Bildebenen deutlich reduziert im Vergleich zu jenem in der Bildebene selbst, weshalb sich diese Technik gut für die Verwendung von Compressed Sensing eignet. Das Konzept ist eng verwandt mit der iterativen Rekonstruktion, wie sie in der CT bereits weitverbreitet angewendet wird. Mit Compressed Sensing kann einerseits die Messzeit deutlich reduziert werden-andererseits kann die Technik auch verwendet werden, um die Stärke der Artefaktkorrektur zu maximieren, beispielsweise, um die Anzahl SEMAC-Schritte zu erhöhen (Abb. 10).

\section{Metallbildgebung bei $3 T$}

Während viele Metallsequenzen schon heute bei $3 \mathrm{~T}$ angewendet werden, bleibt die Bildqualität hinter jener von 1,5T-Geräten zurück. Neue Entwicklungen schaffen hier Abhilfe, sei es der Einsatz von Compressed Sensing oder von lokalen Sende- und Empfangsspulen. Solche Spulen lassen den Einsatz von Radiofrequenzpulsen mit sehr hoher Bandbreite ( $4 \mathrm{kHz}$ ) zu, was eine starke ebenenübergreifende Artefaktkorrektur mit Scan-Zeiten von 7 -9 Minuten ermöglicht (Abb.11) [11].

\section{- Off-Resonance-Suppression (ORS)}

Eine weitere neue Technik ist die sog. Off-ResonanceSuppression (ORS), welche die räumliche Verzerrung oder die Frequenzverzerrung eines angewählten Signals einschränkt und mit anderen Techniken wie VAT, SEMAC oder MAVRIC kombiniert werden kann [12]. 


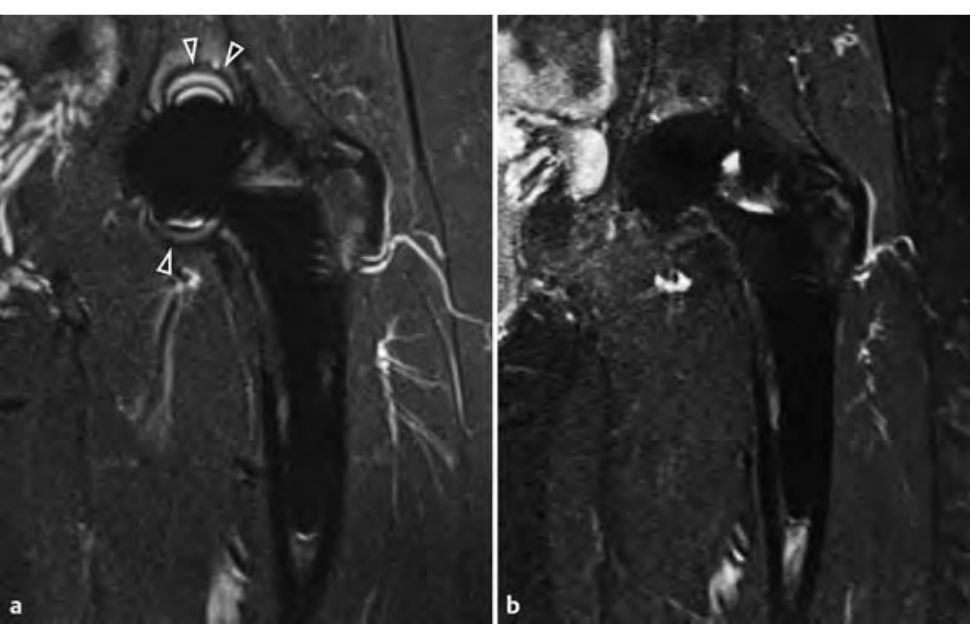

Abb. 10 Artefaktkorrekur bei Hüft-TEP demonstriert an einer koronaren STIR-Sequenz. a Bei der ebenenübergreifenden Artefaktkorrektur mittels SEMAC bleiben teils störende Restartefakte direkt um die Prothesenpfanne (Pfeilspitzen) bestehen, insbesondere, wenn diese aus Kobalt-Chrom besteht. b Wenn SEMAC in Kombination mit Compressed Sensing verwendet wird, können diese Artefakte unterdrückt werden.

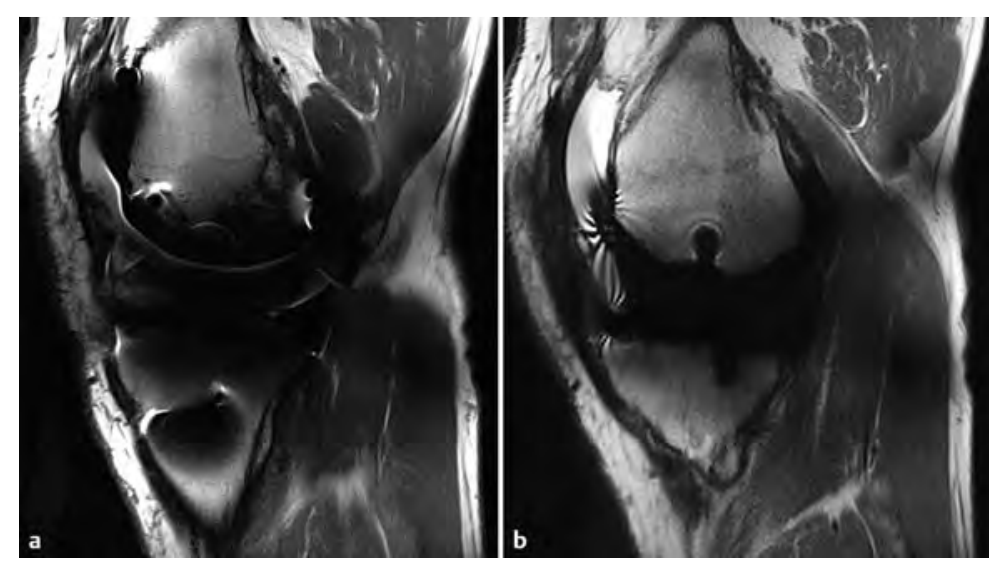

Abb. 11 Artefaktkorrektur bei 3T bei Knie-TEP am Beispiel einer sagittalen protonengewichteten Sequenz und einer lokalen Sende- und Empfangs-Kniespule. a Bei Standardartefaktkorrektur sind bei 3 T große Artefakte um das Metall vorhanden. b Ein Radiofrequenzpuls mit sehr hoher Bandbreite wird zusammen mit der SEMAC-Technik verwendet, was eine sehr starke Artefaktunterdrückung auch bei 3 T ermöglicht.

\section{Metallartefakte in der CT}

\section{Entstehung der Artefakte}

Die 2 hauptsächlichen Verursacher von Metallartefakten in der CT sind die implantatbedingte Absorption der Röntgenphotonen (sog. Photon-,„Starvation“Effekt) und die Aufhärtung des Röntgenstrahlenspektrums (Aufhärtungsartefakte) [13,14]. Weitere Metallartefakt-Komponenten sind akzentuierte Röntgenstrahlen-Streuung durch Richtungsänderung der Röntgenphotonen, Partialvolumeneffekte und Artefakte an Grenzflächen zwischen metallhaltigem Implantat und körpereigenem Gewebe.

\section{- Photon-„Starvation“-Artefakt}

Der Photon-,„Starvation“-Effekt verschlechtert die CT-Bildqualität aufgrund eines Photonenmangels am Detektor, wodurch ein ausgeprägtes Bildrauschen entsteht.

\section{- Aufhärtungsartefakt}

Eine Röntgenröhre emittiert einen polychromatischen Röntgenstrahl, der durch ein breites kontinuierliches Spektrum von niedrig- bis hochenergetischen Photonen charakterisiert ist. Die maximale Photonenenergie wird durch die angelegte Beschleunigungsspannung an der Röntgenröhre begrenzt. Die Photonenenergie wird in Kilo-Elektronenvolt (keV) angegeben, wohingegen die maximal angelegte Beschleunigungsspannung in Kilovolt (kV, engl. „peak kilovoltage“, kVp) quantifiziert wird. Die Röntgenstrahlaufhärtung wird bei der Penetration von Materie beobachtet: Während der Röntgenstrahl ein Objekt durchdringt, werden niederenergetische Photonen (sog. weiche Photonen) häufiger absorbiert (= Photoeffekt) und gestreut (=ComptonStreuung) als höherenergetische Photonen (sog. harte Photonen). Dadurch nimmt die mittlere Photonenenergie zu bzw. verschiebt sich das Röntgenstrahlspektrum hin zu höheren Photonenenergien (=Strahlaufhärtung). Bei Materie mit hoher Elektronendichte wie Knochen oder Metall ist diese Strahlaufhärtung ausgeprägter als bei Weichteilgewebe: Beispielsweise variiert bei einer Becken-CT mit beidseitigen Hüftprothesen die Strahlaufhärtung in Abhängigkeit der verschiedenen Röntgenröhren-Detektor-Winkel innerhalb der Gantry (=Projektionen), sodass hypodense und hyperdense Streifenartefakte entstehen [13].

\section{Metallzusammensetzung}

Das Ausmaß der Metallartefakte wird wesentlich von der Metalllegierung beeinflusst. Implantate aus Titanlegierungen verursachen geringere Artefakte als Kobalt-Chrom-Legierungen und die wiederum geringere Artefakte als Edelstahlimplantate. Dies erklärt, dass z. B. älteres Spondylodesematerial aus Edelstahl stärkere CT-Bildartefakte verursacht als neuere Spondylodese-Implantate aus Titan. Die Absorption der Röntgenstrahlung hängt zudem von der Photonenenergie, der Ordnungszahl eines chemischen Elements und der Massendichte des Materials (=Massenschwächungskoeffizient) ab.

Je tiefer die Photonenenergie in keV, je höher die Massendichte und je größer die Ordnungszahl des chemischen Elements, umso stärker ausgeprägt sind die Metallartefakte [13]. 


\section{Akquisitionsparameter}

\section{Röhrenstrom und Spannung}

Konservative Techniken zur Reduktion von Metallartefakten sind die Erhöhung der Röntgenröhrenspannung und des Röntgenröhrenstroms.

Röhrenspannung. Eine Erhöhung der Röhrenspannung in Kilovolt ( $\mathrm{kV}$, engl. $\mathrm{kVp}$ ) generiert höherenergetische Photonen, die wiederum mit einer größeren Wahrscheinlichkeit das Metallimplantat passieren. Hierdurch können Aufhärtungsartefakte partiell kompensiert werden.

Röhrenstrom. Die Anzahl der Röntgenphotonen erhöht sich linear mit dem Röhrenstrom in MilliampereSekunden (mAs). Je mehr Photonen das Metallimplantat passieren und schließlich am CT-Detektor zum Bildaufbau beitragen, desto geringer ist das Bildrauschen. Damit kann der Photon-,„Starvation“-Effekt partiell korrigiert werden.

Nachteil. Ein direkter Nachteil der Erhöhung der Röntgenröhrenspannung und des Röntgenröhrenstroms ist die Zunahme der Strahlenexposition für den Patienten. Die Röntgenstrahlenexposition steigt mit dem Quadrat der Röhrenspannung und linear mit dem Röhrenstrom. In der Praxis verdoppelt sich die Strahlenexposition mit einem jeweiligen Anstieg der Röhrenspannung um 20 Kilovolt, wohingegen eine Verdoppelung des Röhrenstroms eine doppelt so hohe Strahlenbelastung bedeutet. Somit limitieren strahlenhygienische Aspekte und die technische Leistungsfähigkeit der Röntgenröhre diese beiden konservativen Techniken zur Reduktion von Metallartefakten [13].

\section{- Positionierung des Patienten (Ausrichtung des Metalls)}

Die Dicke des Metallimplantates wirkt sich direkt auf das Ausmaß der Röntgenstrahlenabsorption aus. Zur Reduktion von Metallartefakten sollte daher ein möglichst kleiner Winkel zwischen der Längsrichtung des Metallimplantates und dem Untersuchungstisch gewählt werden (Abb. 12). Limitiert ist dies durch die mögliche Patientenlagerung. Eine Ausrichtung der Implantate parallel zur Längsachse des Untersuchungstischs ist beispielsweise nach Implantation einer Ellenbogenprothese oder Herbert-Schraube im Rahmen einer Skaphoidosteosynthese partiell möglich [13].

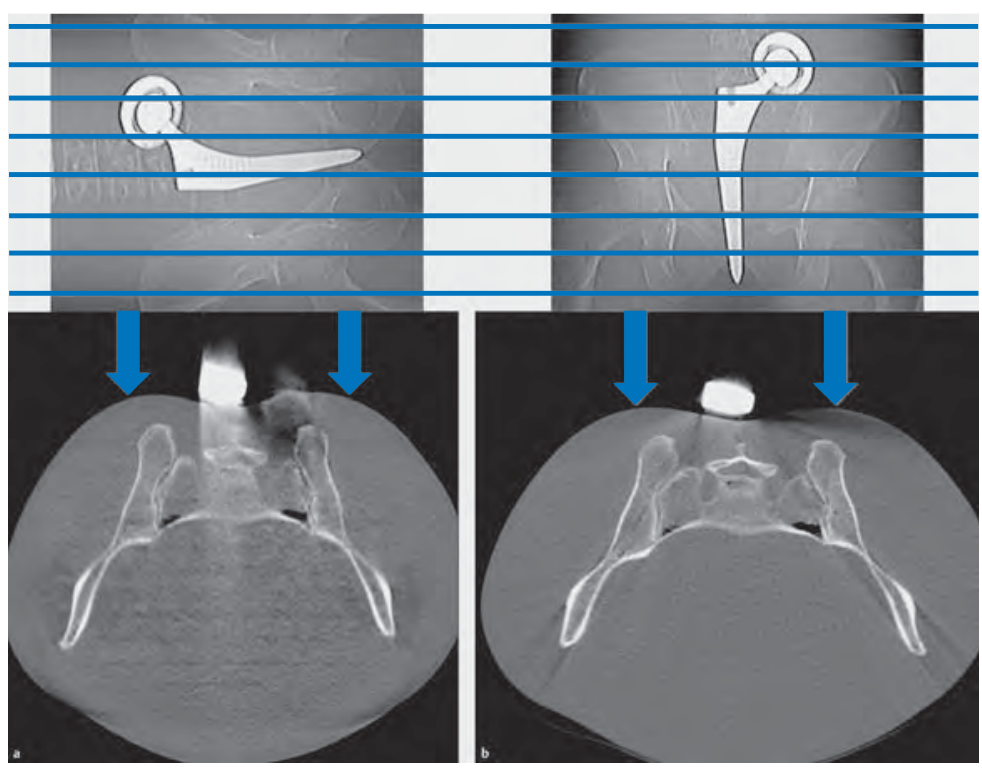

Abb. 12 Die Positionierung des Patienten bzw. die Ausrichtung des Metalls beeinflussen die Metallartefakte. a Eine Hüft-TEP wurde quer zur CT-Tisch-Längsachse auf die Oberfläche des Phantoms gelegt. b Die Hüft-TEP liegt parallel auf dem Phantom. Zur Reduktion von Metallartefakten sollte ein möglichst kleiner Winkel zwischen der Längsrichtung des Metallimplantates und CT-Tisch gewählt werden.

\section{Rekonstruktionsparameter}

\section{- Schichtdicke}

Durch eine Kollimation des Röntgenstrahlfächers bzw. Einblendung der primär akquirierten Schichtdicke z.B. von $1,5 \mathrm{~mm}$ auf $0,75 \mathrm{~mm}$ bei Verwendung eines 16-Zeiler-CTs werden Partialvolumenartefakte sowie Streuartefakte und damit auch Metallartefakte reduziert. Als Konsequenz entsteht ein höheres Bildrauschen. Bildrauschen und Metallartefakte kann man durch die Rekonstruktion dickerer Bildschnitte verringern, beispielsweise bis zu $5 \mathrm{~mm}$ Schichtdicke im Bereich der LWS (Abb. 13). Die Zunahme der rekonstruierten Bildschichtdicke bewirkt, dass die Ortsauflösung abnimmt, wodurch beispielsweise die knöchernen Spongiosabälkchen schwerer abgrenzbar sind [13].

\section{Kernel}

Die Auswahl des Rekonstruktionsfilters (= Kernel) beeinflusst die Metallartefakte maßgeblich.

Ein kantenbetonter Rekonstruktionsfilter (= kantenanhebender Algorithmus, knochenbetonender Kernel) akzentuiert auch die Metallartefakte. Umgekehrt ist die Anwendung glättender Rekonstruktionsfilter vorteilhaft, weil diese auch die Metallartefakte abschwächen (Abb.14). Glättende Rekonstruktionsfilter führen jedoch auch dazu, dass die knöchernen Spongiosabälkchen schwerer abgrenzbar sind. Daher sind Standard- 

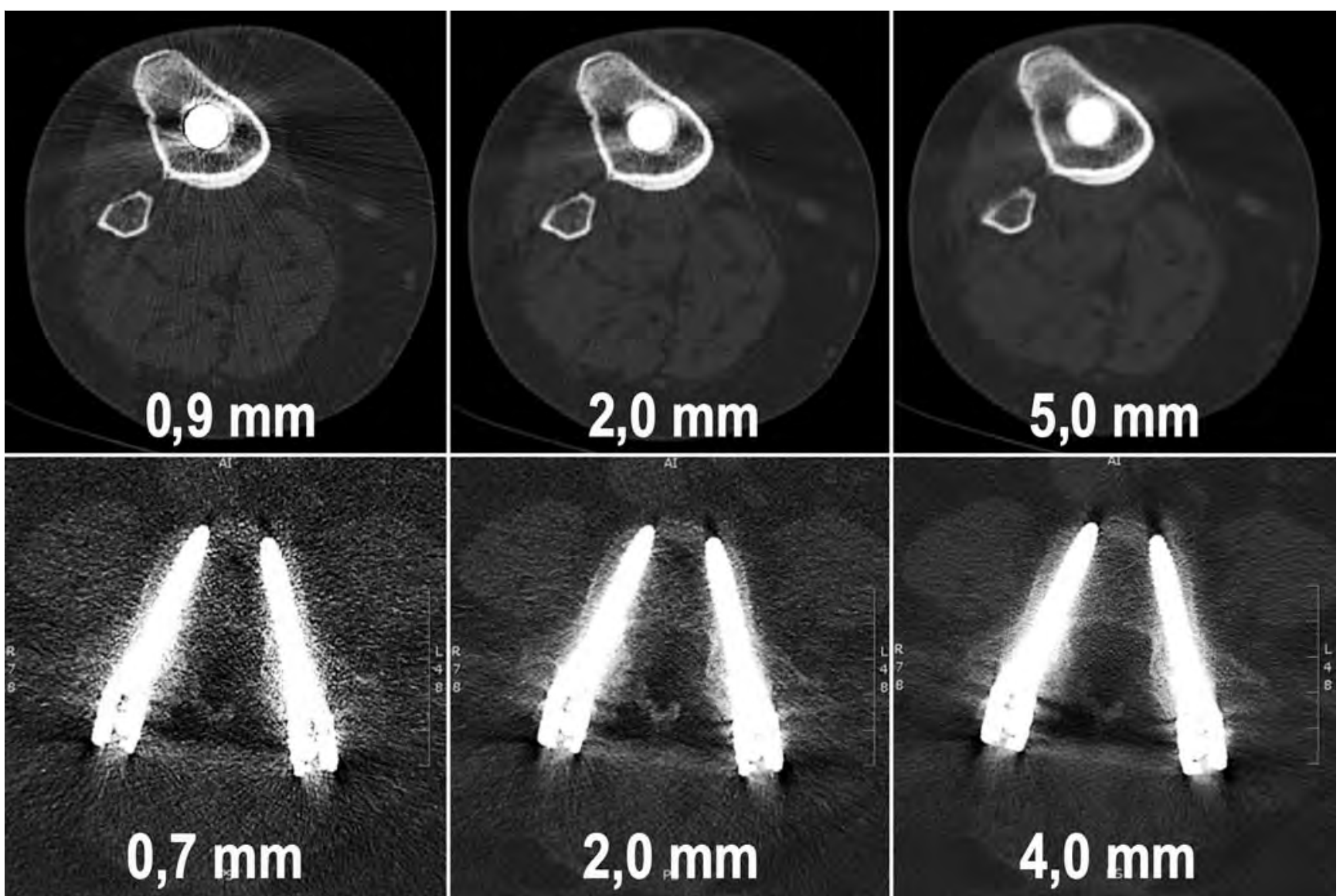

Abb.13 Bildrauschen und Metallartefakte können durch die Rekonstruktion dickerer Bildschnitte reduziert werden, wie hier demonstriert an einer Knieprothese mit Bildern auf Höhe der proximalen Tibia (obere Reihe) bzw. an bilateralen Spondylodeseschrauben der LWS (untere Reihe). Die Erhöhung der rekonstruierten Bildschichtdicke bewirkt eine Abnahme der Ortsauflösung, die in einer erschwerten Abgrenzbarkeit der knöchernen Spongiosabälkchen erkennbar wird.
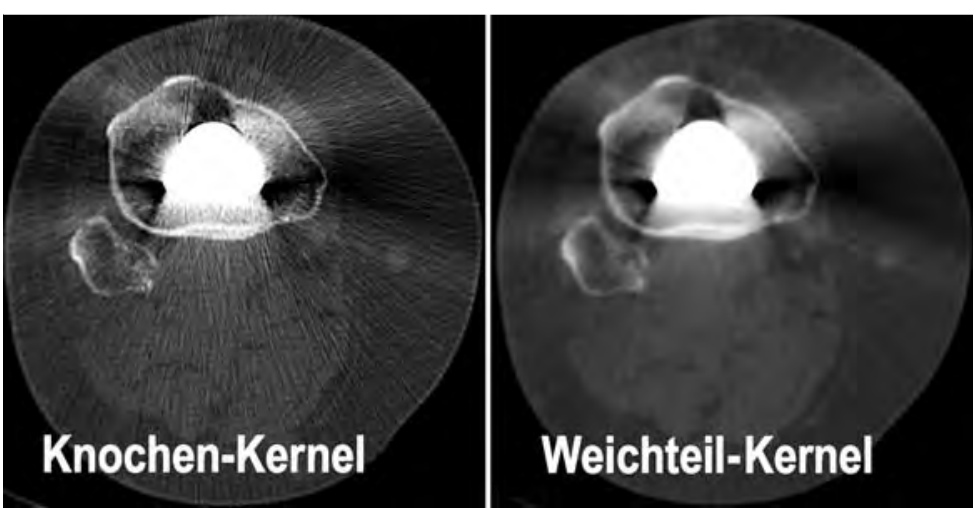

Abb.14 Die Auswahl des Rekonstruktionsfilters (= Kernel) beeinflusst die Metallartefakte. Ein knochenbetonender Kernel ( = kantenanhebender Algorithmus) akzentuiert auch die Metallartefakte (hier demonstriert an einer Knieprothese auf Höhe der proximalen Tibia). Ein glättender Rekonstruktionsfilter reduziert die Metallartefakte und erschwert die Abgrenzbarkeit der knöchernen Spongiosabälkchen. Daher sind Standardrekonstruktionsfilter ( = intermediärer Kernel) zur Reduktion der Metallartefakte ein guter Kompromiss.

rekonstruktionsfilter ( = intermediärer Kernel) zur Reduktion der Metallartefakte ein guter Kompromiss [13].

\section{- Grenzflächen-Artefakte}

Implantat- und Gewebeoberflächen mit großen Hounsfield-Wert-Differenzen resultieren in typischen hypodensen ringförmigen Grenzflächenartefakten, beispielsweise zwischen metallhaltigem Implantat und Knochen wie auch zwischen kortikalem Knochen und angrenzenden Weichteilen. Bei der Auswahl der Rekonstruktionsfilter sollte die Ausprägung dieser Grenzflächenartefakte berücksichtigt werden (Abb.15).

\section{- Erweiterung der Hounsfield-Skala („extended CT scale“)}

Die Standard-Hounsfield-Skala eines CT-Scanners umfasst 4096 Werte $\left(=12 \mathrm{bit},=2^{12}\right)$, beispielsweise von - 1024 Hounsfield-Einheiten (HE) bis 3072 HE. Naturwissenschaftlich betrachtet ist die HE-Skala nach oben offen, insbesondere Metalle weisen eine größere Strahlenabsorption als 3072 HE auf. Einzelne CT-Scanner bieten die Möglichkeit einer erweiterten HE-Skala mit einer maximalen Fensterbreite von 40000 HE. Mittels einer erweiterten HE-Skala können Metallartefakte reduziert werden, beispielsweise bei Positions- 
analysen von Knieprothesen. Nachteilhaft ist dabei die deutliche Reduktion des Weichteilkontrastes [15].

\section{Neuere Techniken}

\section{- Rekonstruktionsalgorithmen}

Iterative Rekonstruktion. Iterative Rekonstruktion basiert auf sich wiederholenden Algorithmen, die das Bild im Anschluss an die Bildakquisition errechnen. Iterative Rekonstruktionstechniken werden zur Reduktion der Strahlendosis eingesetzt. Ein Vergleich zwischen verschiedenen Stärkegraden iterativer Rekonstruktionsalgorithmen und der gefilterten Rückprojektion (Standardmethode) bei Gelenkprothesen ergibt zwar eine offensichtliche Reduktion des Bildrauschens (Abb. 16), das Ausmaß der intrinsischen Metallartefakte wird durch den Einsatz verschiedener Stärkegrade iterativer Standardrekonstruktionsalgorithmen jedoch nicht beeinflusst. Neuere, fortgeschrittene iterative Rekonstruktionsalgorithmen zur Reduktion von Metallartefakten zeigen aber vielversprechende Ergebnisse unter Studienbedingungen. Das Potenzial dieser iterativen Rekonstruktionsalgorithmen zur Reduktion von Metallartefakten muss jedoch außerhalb von Studienbedingungen erst noch der klinische Alltag zeigen.

\section{Projektionsbasierte Artefaktkorrektur. Die meisten} Hersteller bieten eine projektionsbasierte Metallartefaktkorrektursoftware für die klinische Routine an (General Electric: MAR = „smart Metal Artifact Reduction“; Philips: O-MAR = „Metal Artifact Reduction for Orthopedic Implants“, Siemens: iMAR = ,iterative Metal Artifact Reduction“, Toshiba: SEMAR = „Single Energy Metal Artefact Reduction“) [16]. Diese Korrektursoftware basiert auf iterativen Algorithmen, die ausschließlich für die neueren Scanner-Generationen kommerziell verfügbar sind. Gemäß Philips wird während des ersten Schrittes des O-MAR-Algorithmus ein „exklusives Metallbild“ generiert, bei dem allen Weichteilpixeln ein einheitlicher Grauwert zugeordnet wird und die höheren HE-Werte für Metall und Knochen unbeeinflusst bleiben. Anschließend erfolgt u.a. eine komplexe, sich wiederholende Addition und Subtraktion von Ausgangsbild und „exklusivem Metallbild“ zur Reduktion der Metallartefakte.

Damit ermöglicht die projektionsbasierte Artefaktkorrektur (MAR/O-MAR/i-MAR/SEMAR) eine deutliche Reduktion der Metallartefakte in den Weichteilen [16].
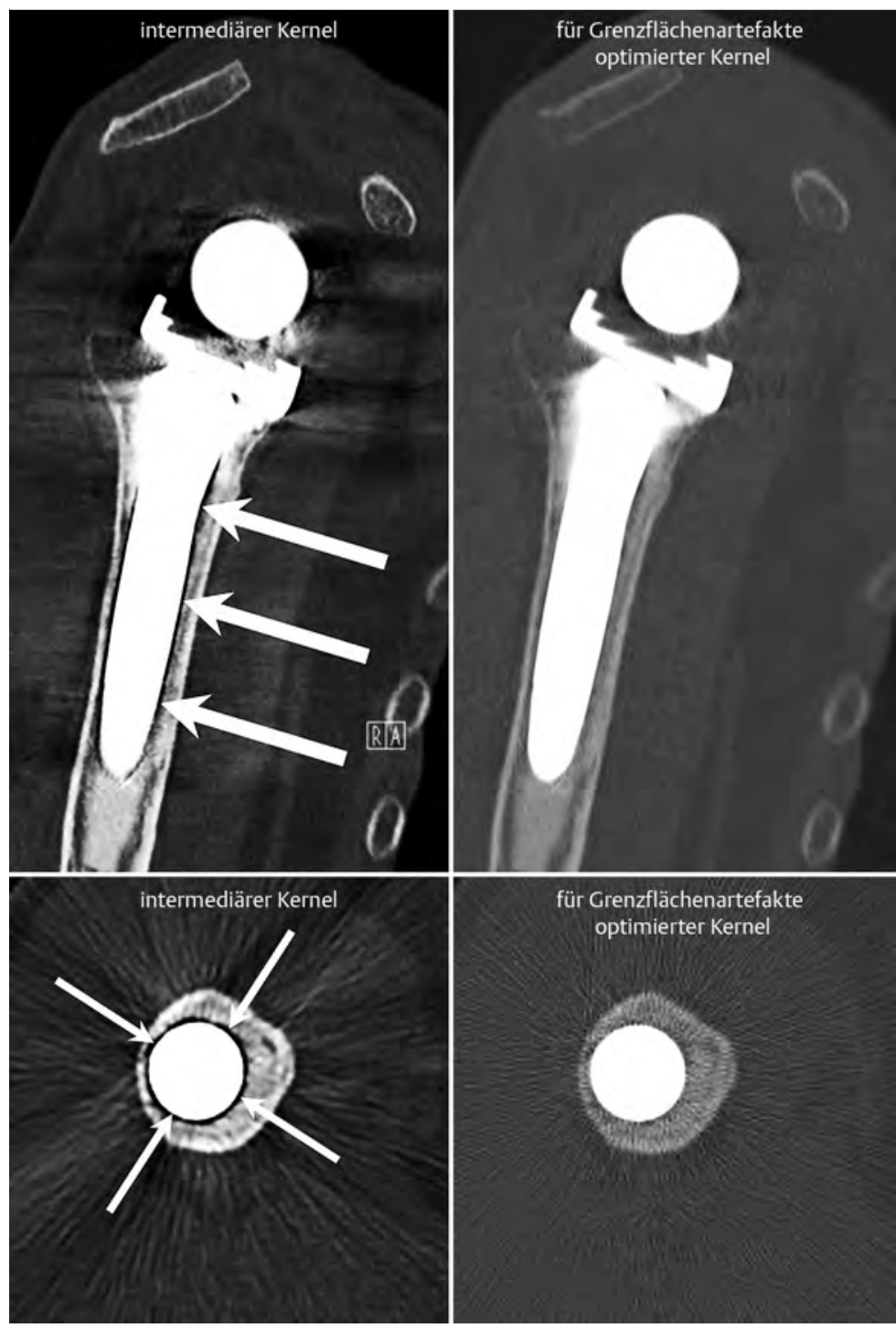

Abb. 15 Hypodense ringförmige Grenzflächenartefakte (Pfeile) entstehen an Implantatund Gewebeoberflächen mit großen Hounsfield-Wert-Differenzen, beispielsweise zwischen der metallhaltigen inversen Schulterprothese und dem Knochenzement/Knochen. Bei der Auswahl der Rekonstruktionsfilter sollte die Ausprägung der Grenzflächenartefakte berücksichtigt werden.

Dies konnte bereits in zahlreichen Studien gezeigt werden. Die projektionsbasierte Artefaktkorrektur kann sowohl für den Radiotherapeuten als auch für den diagnostischen Radiologen hilfreich sein (Abb.17). Die konsekutiv verbesserte Abgrenzbarkeit von Organen und Lymphknoten erleichtert die Beurteilung der Tumorausdehnung. Vorteilhaft für die RadiotherapiePlanung ist auch die höhere Genauigkeit der angegebenen CT-Hounsfield-Werte.

Eingeschränkt wird die diagnostische Beurteilung des Knochens auf den projektionsbasierten MAR-Bildern dadurch, dass regelmäßig Pseudoosteolysen in direkter Nachbarschaft zum Metallimplantat zu sehen sind 

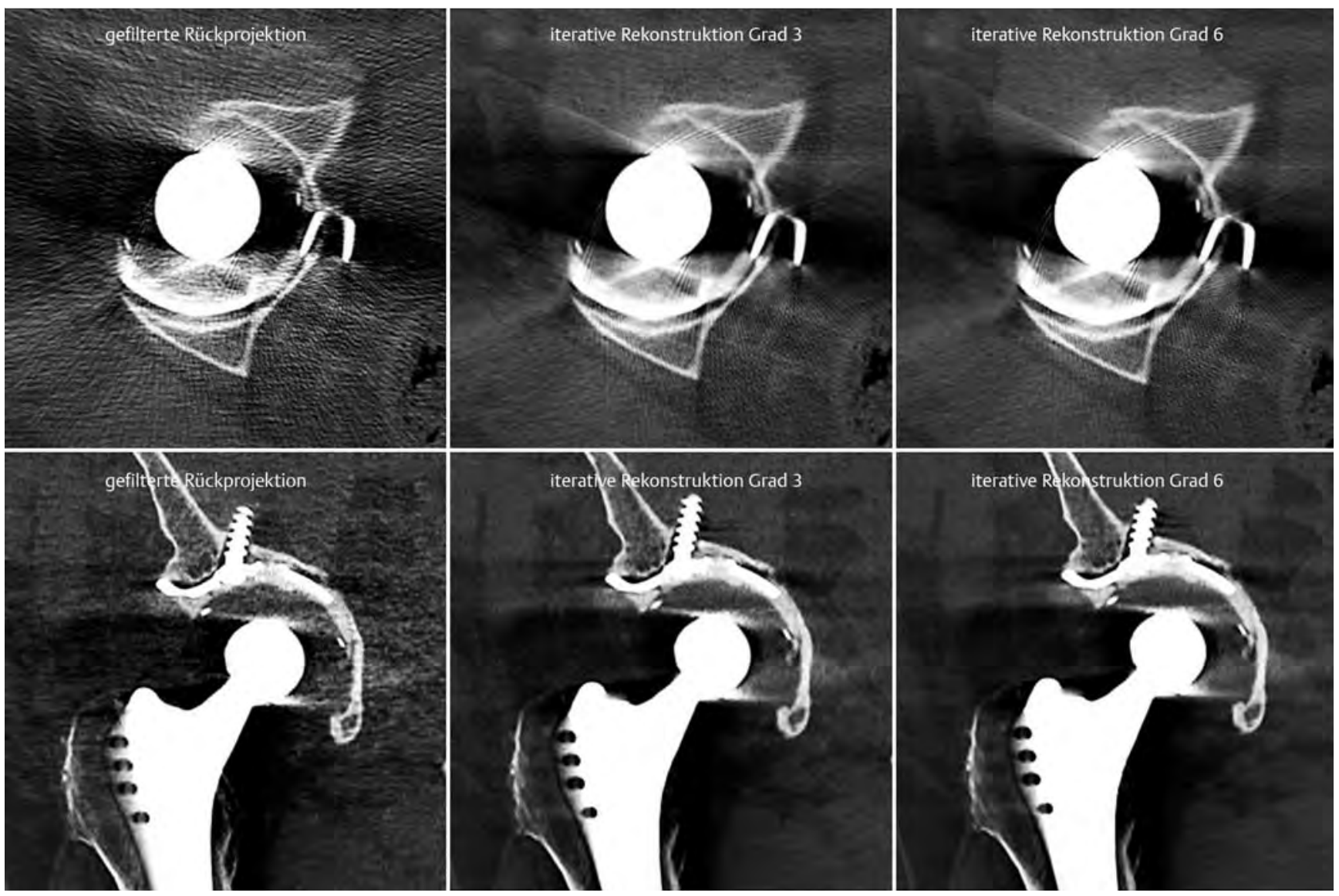

Abb.16 Die Bilder mit iterativen Rekonstruktionsalgorithmen demonstrieren eine Reduktion des Bildrauschens im Vergleich zur gefilterten Rückprojektion bei einem Patienten mit einer Hüft-TEP. Das Ausmaß der intrinsischen Metallartefakte kann durch den Einsatz verschiedener Grade iterativer Standardrekonstruktionsalgorithmen nicht beeinflusst werden.
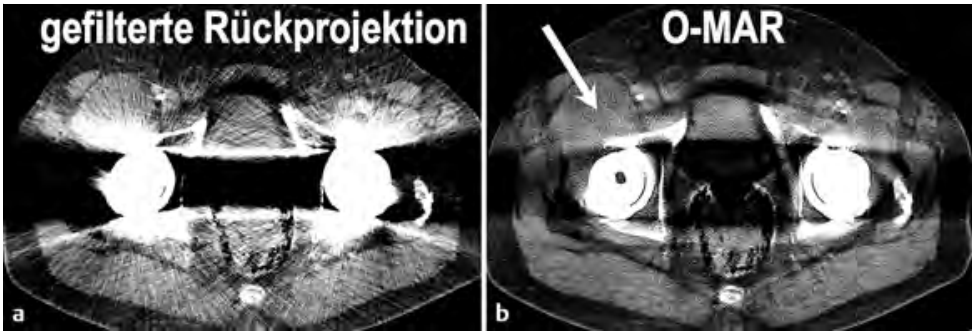

Abb. 17 Die projektionsbasierte Artefaktkorrektur (MAR/O-MAR/i-MAR/SEMAR) ermöglicht eine deutliche Reduktion der Metallartefakte in den zum Metallimplantat und Knochen benachbarten Weichteilen. Durch die deutliche Reduktion der Artefakte im O-MARCT-Bild (b) im Vergleich zur gefilterten Rückprojektion (a) nach beidseitiger Hüft-TEPImplantation sind sowohl die Organe und Lymphknoten im kleinen Becken als auch die stark flüssigkeitsgefüllte rechte Bursa iliopectinea (Pfeil) besser abgrenzbar.

$[16,17]$. Diese Pseudoosteolysen werden u.a. von den zuweisenden Kollegen immer wieder als Implantatlockerung oder auch als Knochenmetastase fehlinterpretiert (Abb. 18). In der orthopädischen Radiologie ist es i.d.R. wichtiger, die Knochensubstanz in der Nachbarschaft des Metallimplantats zu beurteilen als die Metallartefakte in den weiter entfernten Weichteilen zu reduzieren. Daher werden an unserem Institut mit den heute verfügbaren Algorithmen keine projektionsbasierten MAR-Bilder in der klinischen Routine angefertigt.

\section{- Spektrale CT-Bildgebung: virtuelle monochromatische Bildgebung}

Im Unterschied zum mittels Röntgenröhre generierten Röntgenstrahl ist ein monochromatischer Röntgenstrahl aus Photonen gleichförmiger und konstanter Energie zusammengesetzt - es liegt somit eine monoenergetische Strahlung vor. Das Energieniveau dieser monoenergetischen Photonen wird in Kilo-Elektronenvolt (keV) angegeben. Die spektrale CT-Bildgebung mit Dual-Energy-CT-Geräten und detektorbasierten Spektral-CT-Geräten ermöglicht die virtuelle monochromatische Bildgebung [18].

Bei der virtuellen monochromatischen Bildgebung werden CT-Bilder generiert, die auf einem simulierten, theoretischen Röntgenstrahl monoenergetischer Photonen basieren, z. B. $142 \mathrm{keV}$. 
Mittels dieser virtuellen monochromatischen Bildgebung können Aufhärtungsartefakte und konsequenterweise Metallartefakte in der CT-Diagnostik effektiv reduziert werden (Abb.19).

Optimale keV-Werte. Der optimale keV-Wert zur Reduktion von Metallartefakten sollte bei jeder Untersuchung während der Bildnachbearbeitung an der CTArbeitskonsole individuell festgelegt werden. Die Literatur beschreibt für die Reduktion von Metallartefakten ein breites Spektrum an optimalen Werten zwischen $77 \mathrm{keV}$ und $150 \mathrm{keV}[19,20]$. Diese Varianz des optimalen keV-Wertes kann einerseits durch unterschiedliche Durchmesser und Metalllegierungen des Implantates erklärt werden [20]. Andererseits sollte bei der Festlegung des keV-Wertes während der Bildnachbearbeitung berücksichtigt werden, dass niedrigere keV-Werte ein höheres Kontrast-zu-Rausch-Verhältnis und ein niedrigeres Bildrauschen verursachen. Daher sind bei Patienten mit bilateraler Hüft-TEP tiefere monoenergetische Energien, beispielsweise von $77 \mathrm{keV}$, geeignet zur Metallartefaktreduktion im kleinen Becken und konsekutiv besseren Beurteilung der Organe (Genitalorgane, Rektosigmoid, Lymphknoten). Höhere keV-Werte von 120-150 keV eignen sich dagegen, um Metallartefakte im Knochen in Nachbarschaft zum Implantat zu reduzieren und damit periprothetische Osteolysen, Frakturen und Implantatlockerungen besser zu beurteilen.

Strahlenbelastung. Die Strahlenbelastung der spektralen CT-Untersuchungsprotokolle mit dem Ziel der Reduktion von Metallartefakten ist vergleichbar mit der von Standard-CT-Protokollen [14].

Geeignete Geräte. Die meisten für die spektrale CTBildgebung geeigneten Geräte sind die jeweiligen Flaggschiffe der einzelnen Gerätehersteller. Daher sind hohe Investitions- und Unterhaltskosten ein typisches Merkmal von Geräten zur spektralen CT-Bildgebung, was ihre Verbreitung und Anwendung einschränkt. Inzwischen sind jedoch auch preisgünstigere DualEnergy-CT-Geräte auf dem Markt verfügbar, die mit 2 sequenziellen CT-Spiralen von jeweils $80 \mathrm{kV}$ und $140 \mathrm{kV}$ virtuelle monochromatische CT-Bilder ermöglichen [21]. Ein potenzieller Nachteil sind jedoch Bewegungsartefakte aufgrund von Patientenbewegungen zwischen den beiden Spiralen.

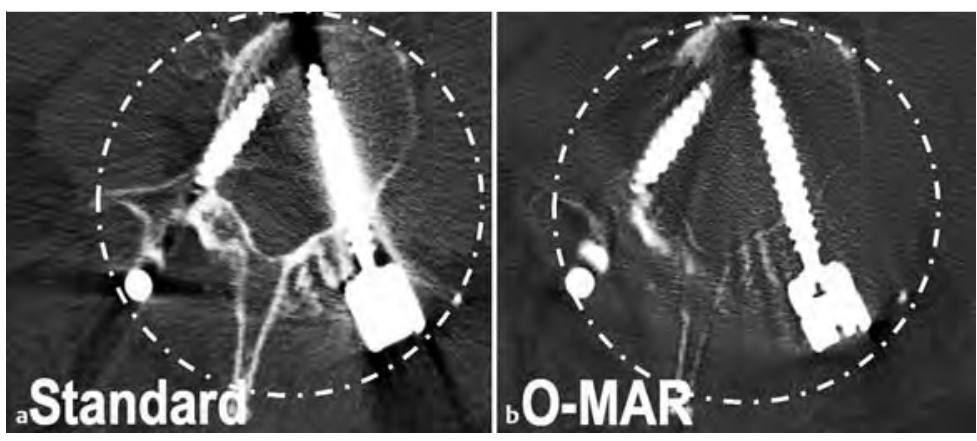

Abb. 18 Projektionsbasierte Bilder zur Reduktion von Metallartefakten (MAR/O-MAR/ i-MAR/SEMAR) generieren regelmäßig Pseudoosteolysen in Nachbarschaft zum Metallimplantat aufgrund des bei der Bildrekonstruktion verwendeten Algorithmus, hier z. B. bei einem Patienten mit Spondylodeseschrauben an der LWS. An identischer Position sind im Vergleich zur Standardrekonstruktion (a) ausgedehnte Pseudoosteolysen in der O-MARRekonstruktion (b) sichtbar. Neben den Metallartefakten sind im O-MAR-Bild große Areale mit normaler Knochenstruktur verschwunden. Pseudoosteolysen können als Implantatlockerung oder osteolytische Neoplasie fehlinterpretiert werden.

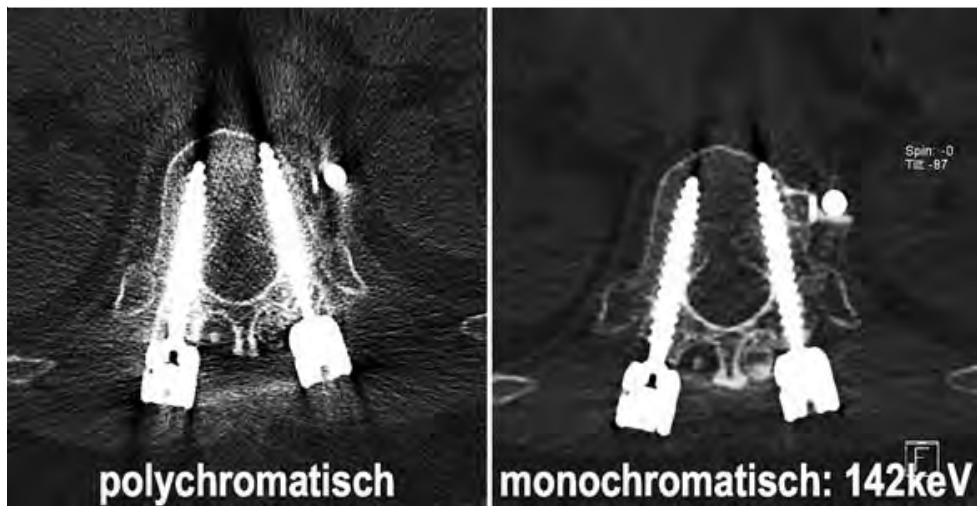

Abb.19 Die virtuelle monochromatische Bildgebung, z. B. 142 keV, mittels Dual-EnergyCT-Geräte und detektorbasierter Spektral-CT-Geräte kann Aufhärtungsartefakte des Metalls effektiv reduzieren.

\section{Klinische Bedeutung der MRT und $\mathrm{CT}$}

\section{MRT}

Noch vor 15 Jahren galt die MRT als nicht geeignet für die Untersuchung von Patienten mit Gelenkprothesen, da die ausgeprägten Artefakte häufig eine diagnostische Bildqualität verhinderten. In der Zwischenzeit hat diesbezüglich ein radikales Umdenken stattgefunden. Entscheidend hierfür war die Verbesserung der MRTTechnik, mit der es möglich wurde, die Weichteile und später auch den Knochen um Gelenkprothesen sowie die Gelenkkapsel mit moderaten oder teilweise fast ohne Artefakte darzustellen.

Prothesen werden heute bei Arthrosen verschiedener Gelenke häufig eingesetzt. Meist führt eine solche Operation zu einem zufriedenstellenden Resultat. Eine 
Minderheit der Patienten hat jedoch auch nach dem Einsatz eines künstlichen Gelenks Restbeschwerden. Während die MRT nicht zur initialen Abklärung solcher Patienten verwendet wird, hat sie nach erfolgter klinischer Untersuchung sowie der konventionellen radiologischen Abklärung einen wichtigen Stellenwert bei Patienten mit unklaren Restbeschwerden. Die MRT erlaubt z. B. den Nachweis knöcherner Stressreaktionen um Gelenkprothesen oder um Spondylodeseschrauben an der Wirbelsäule und kann auch die Lockerung eines Metallimplantates nachweisen. Auch Weichteilabnormitäten wie Risse der glutealen Sehneninsertionen bei Hüftprothesen werden zuverlässig dargestellt. Seit dem gehäuften Auftreten von Pseudotumoren und Metallose-assoziierten Osteolysen bei Patienten mit MetallMetall-Gleitpaarung nach Hüftgelenkersatz ist die MRT für diese Patienten zu einer wichtigen Stütze für das Erkennen materialassoziierter Komplikationen geworden. Zudem werden invasive Untersuchungen durch die MRT ersetzt: Bei Patienten mit viel Spondylodesematerial wurden früher regelmäßig CT-Myelografien mit Kontrastmittelinjektion in den Liquor durchgeführt, um Diskushernien und Spinalkanalstenosen zu erkennen. Dank der modernen MRT-Bildgebung kann man Patienten mit Spondylodesen diese invasive diagnostische Untersuchung nun meist ersparen.

\section{CT}

Im Unterschied zur MRT wird die CT schon seit Längerem eingesetzt, um Gelenkprothesen und orthopädische Implantate zu beurteilen. Während die MRT teils kontraindiziert ist und die Patienten über 20 30 Minuten ruhig im Gerät liegen müssen, hat die CT den großen Vorteil, dass sie bei fast allen Patienten angewendet werden kann. Die Untersuchungszeit für die CT beträgt mit Planung typischerweise 2-3 Minuten, wobei allerdings die Zeit für die Nachrekonstruktion der akquirierten Daten ein Mehrfaches davon betragen kann.

Die CT hat bei Patienten mit orthopädischen Implantaten ein breites Indikationsspektrum, wie z. B. den Nachweis einer Lockerung oder eines Materialbruchs von Spondylodeseschrauben, von schmalen Zonen der ossären Durchbauung bei Spondylodesen oder von periprothetischen Frakturen.

\section{Wahl des Verfahrens}

Welche Modalität im konkreten Fall angewendet wird, hängt von der lokal vorhandenen Expertise und den technischen Möglichkeiten ab. Da verschiedene Techniken sowohl bei der MRT als auch der CT noch mitten

\section{Kernaussagen}

- Der menschliche Körper besteht aus einer Vielzahl von Gewebearten, die eine unterschiedliche Magnetisierbarkeit, die sog. magnetische Suszeptibilität aufweisen. Am stärksten verändert wird das Magnetfeld dabei durch ferromagnetische Substanzen.

- Grundtypen von Metallartefakten in der MRT sind Dephasierung mit Verlust oder Akkumulation von Bildsignal, geometrische Distorsion sowie ungenügende Fettunterdrückung.

- MRT-Metallartefakte können aus der Bildebene selbst stammen (In-PlaneArtefakte) oder aus den benachbarten Bildebenen (Through-Plane-Artefakte).

- Da ein direkter Zusammenhang zwischen Feldstärke und Artefaktgröße besteht, ist für die MRT-Metallbildgebung eine magnetische Flussdichte von 1,5 T gegenüber $3 \mathrm{~T}$ klar im Vorteil.
- Einfache Methoden zur MRT-Artefaktkorrektur sind die Erhöhung der Empfängerbandbreite, die Wahl einer kleinen Schichtdicke und Voxelgröße sowie der Einsatz von Turbo-Spin-EchoSequenzen.

- Die STIR-Sequenz erlaubt eine deutlich homogenere Fettunterdrückung in der MRT als die spektrale Fettsaturierung, insbesondere wenn ein optimierter Inversionspuls zur Anwendung kommt.

- Fortgeschrittene Methoden zur MRTArtefaktkorrektur sind die Dixon-Technik, das View-Angle-Tilting sowie die ebenenübergreifende Artefaktkorrektur (SEMAC, MAVRIC).

- Die 2 hauptsächlichen Verursacher von CT-Metallartefakten sind die Absorption der Röntgenphotonen sowie Aufhärtungsartefakte.

- Konservative Techniken zur Reduktion von CT-Metallartefakten sind die Erhö- hung der Röntgenröhrenspannung wie auch des Röntgenröhrenstroms, was allerdings mit einer erhöhten Strahlendosis einhergeht.

- Eine dünnere Kollimation sowie eine anschließende Rekonstruktion dickerer Bildschnitte reduziert CT-Metallartefakte.

- Die projektionsbasierte Artefaktkorrektur (MAR/O-MAR/i-MAR/SEMAR) ermöglicht eine deutliche Reduktion der Metallartefakte in den Weichteilen. Diese Methoden führen jedoch regelmäßig zu Pseudoosteolysen in direkter Nachbarschaft zum Metallimplantat, was eine diagnostische Beurteilung des Knochens stark einschränkt.

- Die spektrale CT-Bildgebung mit virtueller monochromatischer Röntgenstrahlung erlaubt, Aufhärtungsartefakte zu vermeiden. 
in der Entwicklung sind, ist mit Änderungen des Indikationsspektrums zu rechnen. Wichtig ist, mit den orthopädischen Kollegen das direkte Gespräch zu suchen, damit Radiologen und Orthopäden einen Konsens finden können, was die Fragestellungen, die geeigneten Modalitäten und ihre Grenzen angeht.

\begin{abstract}
Joint replacement and other orthopaedic implants are utilized in many patients with musculoskeletal disorders. While these operations commonly show a good clinical result, a substantial number of patients need to undergo postoperative imaging during follow-up. The presence of orthopaedic implants induces severe metal artefacts at MRI and CT. We review several basic methods and advanced techniques for reducing metal artefacts at MRI and CT in order to enable a diagnostic examination in patients with metal implants. With the use of these techniques, MRI and CT are important and reliable modalities to examine patients with joint replacement and orthopaedic implants.
\end{abstract}

\section{Keywords}

Metal artefacts · joint replacement - implants . musculoskeletal imaging $\cdot \mathrm{MRI} \cdot \mathrm{CT}$

\section{Über die Autoren}

\section{Reto Sutter}

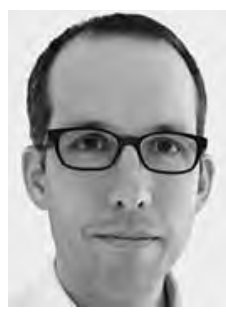

Priv.-Doz. Dr. med. Studium der Humanmedizin an der Universität Zürich. Promotion an der Universität Zürich. Facharztausbildung Radiologie: Diagnostische Radiologie Universitätsspital Zürich, Neuroradiologie Universitätsspital Zürich, Pathologie Royal London Hospital und Fellowship Neurowissenschaften Queen Mary University London/UK, Radiologie Kantonsspital Winterthur. Oberarzt Radiologie Kantonsspital Winterthur. Einjähriges Fellowship muskuloskelettale Radiologie, orthopädische Universitätsklinik Balgrist Zürich. Mehrjähriger Oberarzt und Leitender Arzt Radiologie, orthopädische Universitätsklinik Balgrist Zürich. Europäisches Diplom für Muskuloskelettale Radiologie (ESSR). Habilitation an der Universität Zürich. Seit 2016 Stellvertretender Chefarzt Radiologie, orthopädische Universitätsklinik Balgrist Zürich.

\section{Tobias Dietrich}

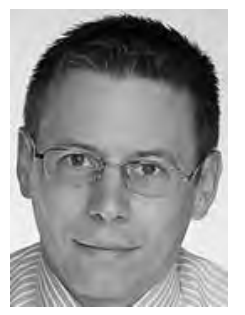

Priv.-Doz. Dr med. Medizinstudium und Promotion an der EberhardKarls-Universität Tübingen. Facharztausbildung Radiologische Diagnostik: Universitätsklinikum Tübingen (D), Radioonkologie Universitätsklinikum Tübingen (D), Radiologie Kantonsspital Winterthur $(\mathrm{CH})$ und Diagnostische Radiologie Universitätsspital Zürich (CH). Oberarzt Radiologie Kantonsspital Münsterlingen ( $\mathrm{CH}$ ). Einjähriges Fellowship Muskuloskelettale Radiologie und anschließend mehrjähriger Oberarzt Radiologie orthopädische Universitätsklinik Balgrist, Zürich (CH). Seit Juni 2016 Leitung Muskuloskelettale Radiologie Kantonsspital St. Gallen (CH). Habilitation Universität Zürich. Spezialisierung: Sportverletzungen des Bewegungsapparates. Orthopädische und rheumatologische Bildgebung. Minimal invasive Schmerztherapie an der Wirbelsäule und des Bewegungsapparates.

\section{Korrespondenzadresse}

Priv.-Doz. Dr. med. Reto Sutter

Radiologie

Universitätsklinik Balgrist

Forchstraße 340

8008 Zürich/Schweiz

Tel. +41443863308

Fax: +41443863319

E-Mail: reto.sutter@balgrist.ch

Interessenkonflikt: kein Interessenkonflikt angegeben

\section{Literatur}

1 Beswick AD, Wylde V, Gooberman-Hill R et al. What proportion of patients report long-term pain after total hip or knee replacement for osteoarthritis? A systematic review of prospective studies in unselected patients BMJ open 2012; 2: e000435

2 Miller TT. Imaging of joint replacements. Seminars in musculoskeletal radiology 2015; 19: 1 -2

3 Hargreaves BA, Worters PW, Pauly KB et al. Metal-induced artifacts in MRI. AJR 2011; 197: 547 - 555

4 Liebl $\mathrm{H}$, Heilmeier $\mathrm{U}$, Lee $\mathrm{S}$ et al. In vitro assessment of knee MRI in the presence of metal implants comparing MAVRIC-SL and conventional fast spin echo sequences at 1.5 and $3 \mathrm{~T}$ field strength. J Magn Reson Imaging 2014

5 Ulbrich EJ, Sutter R, Aguiar RF et al. STIR sequence with increased receiver bandwidth of the inversion pulse for reduction of metallic artifacts. AJR Am J Roentgenol 2012; 199: W735- 742 
6 Rampton JW, Young PM, Fidler JL et al. Putting the fat and water protons to work for you: a demonstration through clinical cases of how fat-water separation techniques can benefit your body MRI practice. AJR Am J Roentgenol 2013; 201: $1303-1308$

7 Sutter R, Ulbrich EJ, Jellus V et al. Reduction of Metal Artifacts in Patients with Total Hip Arthroplasty with Slice-encoding Metal Artifact Correction and View-Angle Tilting MR Imaging. Radiology 2012; 265: 204-214

8 Koch KM, Brau AC, Chen W et al. Imaging near metal with a MAVRIC-SEMAC hybrid. Magn Reson Med 2011; 65: 71-82

9 Sutter R, Stoel BC, Buck FM et al. Internal Derangements of Joints-Past, Present, and Future. Invest Radiol 2015; 50: 601 614

10 Worters PW, Sung K, Stevens KJ et al. Compressed-sensing multispectral imaging of the postoperative spine. J Magn Reson Imaging 2013; 37: $243-248$

11 Bachschmidt TJ, Sutter R, Jakob PM et al. Knee implant imaging at 3 Tesla using high-bandwidth radiofrequency pulses. J Magn Reson Imaging 2015; 41: 1570-1580

12 den Harder JC, van Yperen GH, Blume UA et al. Off-resonance suppression for multispectral MR imaging near metallic implants. Magn Reson Med 2015; 73: 233-243

13 Lee MJ, Kim S, Lee SA et al. Overcoming artifacts from metallic orthopedic implants at high-field-strength MR imaging and multi-detector CT. Radiographics 2007; 27: 791-803

14 Pessis E, Campagna R, Sverzut JM et al. Virtual monochromatic spectral imaging with fast kilovoltage switching: reduction of metal artifacts at CT. Radiographics 2013; 33: 573-583
15 Link TM, Berning W, Scherf S et al. CT of metal implants: reduction of artifacts using an extended $\mathrm{CT}$ scale technique. J Comput Assist Tomogr 2000; 24: 165-172

16 Gondim Teixeira PA, Meyer JB, Baumann C et al. Total hip prosthesis $C T$ with single-energy projection-based metallic artifact reduction: impact on the visualization of specific periprosthetic soft tissue structures. Skeletal Radiol 2014; 43: $1237-1246$

17 Huang JY, Kerns JR, Nute JL et al. An evaluation of three commercially available metal artifact reduction methods for CT imaging. Phys Med Biol 2015; 60: 1047-1067

18 Guggenberger R, Winklhofer S, Osterhoff G et al. Metallic artefact reduction with monoenergetic dual-energy $\mathrm{CT}$ : systematic ex vivo evaluation of posterior spinal fusion implants from various vendors and different spine levels. Eur Radiol 2012; 22: 2357-2364

19 Han SC, Chung YE, Lee YH et al. Metal artifact reduction software used with abdominopelvic dual-energy $\mathrm{CT}$ of patients with metal hip prostheses: assessment of image quality and clinical feasibility. AJR Am J Roentgenol 2014; 203: 788-795

20 Bamberg F, Dierks A, Nikolaou K et al. Metal artifact reduction by dual energy computed tomography using monoenergetic extrapolation. Eur Radiol 2011; 21: 1424-1429

21 Mangold S, Gatidis S, Luz O et al. Single-source dual-energy computed tomography: use of monoenergetic extrapolation for a reduction of metal artifacts. Invest Radiol 2014; 49: $788-793$ 


\section{CME-Fragen}

Die folgenden Fragen beziehen sich auf den vorangehenden Beitrag. Bitte schicken Sie uns die entsprechenden Lösungsbuchstaben. Jeweils eine Antwort ist richtig. Die Vergabe von CME-Punkten ist an die korrekte Beantwortung der Multiple-Choice-Fragen gebunden.

1

Welche der folgenden Aussagen ist richtig? Ein von außen angelegtes Magnetfeld ...?
A wird durch den kortikalen Knochen leicht verstärkt.
B wird durch Titan leicht verstärkt.
C wird durch Wasser verstärkt.
D wird durch gadoliniumhaltige Kontrastmittel leicht abgeschwächt.
E wird durch Nickel abgeschwächt.

\section{2}

Wodurch entstehen die stärksten Metallartefakte in der MRT?
A Metallabrieb nach Arthroskopie
B Sehnenanker der Rotatorenmanschette
C Knie-Prothese
D Plattenosteosynthese
E abgebrochene Bohrerspitze im Knochen

\section{3}

Welche der folgenden Aussagen ist richtig?
A MRT-Metallartefakte nehmen mit zunehmender Feldstärke zu.
B MRT-Metallartefakte nehmen mit zunehmendem Signal-zu-Rausch-Verhältnis zu.
C MRT-Metallartefakte nehmen mit zunehmender Empfängerbandbreite zu.
D MRT-Metallartefakte nehmen mit zunehmender Schichtdicke ab.
E MRT-Metallartefakte nehmen mit zunehmender Fettunterdrückung zu.

Welche Technik ist nicht geeignet, um MRT-Metallartefakte zu reduzieren?
A Dixon-Technik
B spektrale Fettsaturierung
C View-Angle-Tilting
D SEMAC
E STIR mit optimiertem Inversionspuls

Was ist keine Ursache für CT-Metallartefakte?
A Opposed-Phase-Imaging
B In-Phase-Imaging
C Optimized-Bandwidth-Correction
D Through-Plane-Correction
E Field-Strength-Correction

6
A Absorption der Röntgenphotonen
B Aufhärtung des Röntgenstrahlenspektrums
C Röntgenstrahlen-Streuung
D Paarbildung
E Artefakte an Grenzflächen zwischen dem metallhaltigen Implantat und körpereigenen Gewebe 


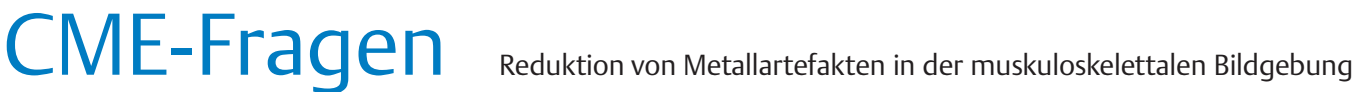

\section{7}

Welche der folgenden Regeln ist richtig, um Metallartefakte in der CT-Diagnostik zu reduzieren?
A Installation des CT-Gerätes in einem Faraday-Käfig
B suffiziente Bleiabdeckung des Patienten
C Ausrichtung des Metalls parallel zur Tischlängsachse
D ansprechende vielfarbige Beleuchtung des Schaltraums
E schnelle Bildgebung mittels Pitch-Erhöhung

\section{8}

Welche der folgenden Regeln ist richtig in Bezug auf die Verdoppelung der Röntgenstrahlenexposition für den Patienten?
A Anstieg der Röhrenspannung um jeweils $20 \mathrm{kV}$
B Anstieg des Röhrenstroms um jeweils $20 \mathrm{mAs}$
C Die Röntgenstrahlenexposition steigt mit dem Quadrat des Röhrenstroms.
D Die Schichtdicke und Strahlenexposition stehen invers linear zueinander.
E Eine Verdoppelung der Strahlenexposition halbiert das Bildrauschen.

9

Welche der folgenden Aussagen zur Reduktion von Metallartefakten in der CT-Diagnostik ist richtig?
A Iterative Rekonstruktionsalgorithmen sind immer wirkungsvoll in der Reduktion von Metallartefakten.
B Ein Dual-Energy-CT-Gerät ermöglicht eine wirkungsvolle Reduktion der Metallartefakte.
C Aufhärtungsartefakte werden durch eine niedrige Beschleunigungsspannung verhindert.
D Ein moderner CT-Scanner generiert automatisch Bilder mit einer maximalen Reduktion der Metallartefakte.
E Metallhaltige Implantate sind eine klassische Kontraindikation für eine CT-Untersuchung.

Welche der folgenden Aussagen zur projektionsbasierten Korrektur von Metallartefakten (MAR/O-MAR/ i-MAR/SEMAR) in der (T-Diagnostik ist richtig?
A Pseudoosteolysen sind kein Problem.

B Es werden Metallartefakte in den Weichteilen in Nachbarschaft der Metallimplantate reduziert.

C Diese Technologie wird seit mehreren Jahrzehnten in allen handelsüblichen CT-Scannern angewendet.

D In der Radiotherapie-Planung ist diese Technik bedeutungslos.

E Die alleinige Rekonstruktion von projektionsbasierten Metallartefaktkorrektur-CT-Bildern ist ausreichend.

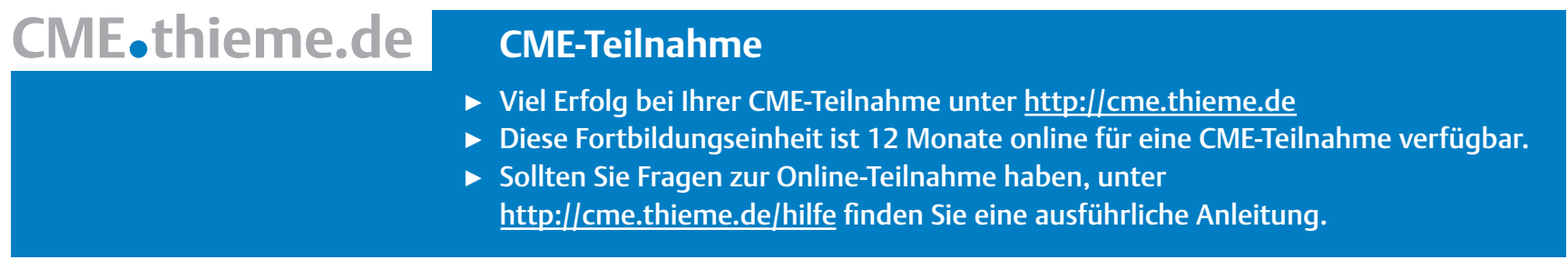

